\title{
BLOWING UP EXTREMAL KÄHLER MANIFOLDS II
}

\author{
GÁBOR SZÉKELYHIDI
}

\begin{abstract}
This is a continuation of the work of Arezzo-Pacard-Singer and the author on blowups of extremal Kähler manifolds. We prove the conjecture stated in [32], and we relate this result to the K-stability of blown up manifolds. As an application we prove that if a Kähler manifold $M$ of dimension greater than 2 admits a cscK metric, then the blowup of $M$ at a point admits a cscK metric if and only if it is $\mathrm{K}$-stable, as long as the exceptional divisor is sufficiently small.
\end{abstract}

\section{INTRODUCTION}

We continue our study [32] of extremal metrics on blown-up manifolds, following the work of Arezzo-Pacard [1, 2] and Arezzo-Pacard-Singer [3. See Pacard 21] for a survey and see also LeBrun-Singer [18, Rollin-Singer [23], Tipler [35, BiquardRollin 4] for related work. The starting point is a compact Kähler manifold $M$ with an extremal metric $\omega$. The notion of extremal metric was introduced by Calabi [6], and it means that the gradient of the scalar curvature $\nabla \mathbf{s}(\omega)$ is a holomorphic vector field. The basic question that we study is whether the blowup $\mathrm{Bl}_{p_{1}, \ldots, p_{n}} M$ of $M$ in a finite number of points admits an extremal metric in the Kähler class

$$
\pi^{*}[\omega]-\varepsilon^{2}\left(a_{1}\left[E_{1}\right]+a_{2}\left[E_{2}\right]+\ldots a_{n}\left[E_{n}\right]\right),
$$

where $\pi$ is the blowdown map, $a_{1}, \ldots, a_{n}>0$ are constants, $\varepsilon>0$ is very small, and $E_{i}$ are the exceptional divisors. Our methods, following [1, 2, 3] are perturbative, restricting the results to sufficiently small $\varepsilon>0$. In addition our results will be restricted to blowing up only one point, and dimension $m>2$. We expect that with some more work our method can deal with the case $m=2$, but blowing up more than one point introduces more serious difficulties as we will explain in Section 3.2

To state the main result, let us write $G$ for the group of Hamiltonian isometries of $(M, \omega)$ and $\mathfrak{g}$ for its Lie algebra. Let

$$
\mu: M \rightarrow \mathfrak{g}^{*}
$$

be the equivariant moment map for the action of $G$ normalized in such a way that the Hamiltonian functions $\langle\mu, \xi\rangle$ have zero mean on $M$ for all $\xi \in \mathfrak{g}$. From now on we will identify $\mathfrak{g}$ with its dual, using the inner product given by the $L^{2}$ product on Hamiltonian functions. Let $\Delta \mu$ be the Laplacian of $\mu$ taken componentwise after identifying $\mathfrak{g}^{*}$ with $\mathbf{R}^{l}$ for some $l$. A central role is played by the perturbed moment map

$$
\mu(p)+\delta \Delta \mu(p)
$$

for small $\delta$. Note that this is simply the moment map for the action of $G$ on $M$ with respect to the Kähler form $\omega-\delta \rho$, where $\rho$ is the Ricci form of $\omega$. Let us write $G^{c}$ for the complexification of $G$, acting on $M$ by biholomorphisms. With this the main result is as follows, confirming Conjecture 6 in [32 in the case when $m>2$. 
Theorem 1. Assume that the dimension $m>2$, and suppose that $\nabla \mathbf{s}(\omega)$ vanishes at $p \in M$. There is a $\delta_{0}>0$ depending on $(M, \omega)$ with the following property. Suppose that for some $\delta \in\left(0, \delta_{0}\right)$ there is a point $q$ in the $G^{c}$-orbit of $p$ such that the vector field $\mu(q)+\delta \Delta \mu(q)$ vanishes at $q$. Then the blowup $\mathrm{Bl}_{p} M$ admits an extremal metric in the Kähler class

$$
\pi^{*}[\omega]-\varepsilon^{2}[E],
$$

for all sufficiently small $\varepsilon>0$.

Suppose that $M$ is a projective variety and $\omega \in c_{1}(L)$ for a line bundle $L$ over $M$. The condition in the theorem can be interpreted as relative stability of the point $p$ with respect to the natural linearization of the $G^{c}$-action on the $\mathbf{Q}$-line bundle $L+\delta K_{M}$ for small rational $\delta$, where $K_{M}$ is the canonical bundle. In this terminology, our earlier result in 32 only dealt with the case when $p$ is relatively stable with respect to the linearization on $L$. This in turn refined earlier results of Arezzo-Pacard-Singer [3], where some extra conditions were required. Allowing a small perturbation of the line bundle $L$ gives more precise information about case when the point $p$ is strictly semistable.

In the case when $(M, \omega)$ is a constant scalar curvature Kähler (or cscK) manifold, then we can show that Theorem 10 actually gives a complete characterization of the possible blowup points. Suppose again that $M$ is projective and $\omega \in c_{1}(L)$. For small rational $\varepsilon$ let us write $L_{\varepsilon}=\pi^{*} L-\varepsilon^{2}[E]$ for an ample $\mathbf{Q}$-line bundle on the blowup $\mathrm{Bl}_{p} M$. The Yau-Tian-Donaldson conjecture [37, 34, 11] predicts that the existence of a cscK metric on the blowup $\mathrm{Bl}_{p} M$ in the first Chern class $c_{1}\left(L_{\varepsilon}\right)$ is related to the K-stability of the pair $\left(\mathrm{Bl}_{p} M, L_{\varepsilon}\right)$. In Section 4 we define a simple version of K-stability for Kähler manifolds, restricting attention to testconfigurations with smooth central fibers. Using this and Theorem 1 we obtain the following.

Theorem 2. Let $(M, \omega)$ be a cscK manifold of dimension $m>2$, and let $p \in M$. Then for sufficiently small $\varepsilon>0$ the following are equivalent, and are independent of $\varepsilon$ :

(1) The blowup $\mathrm{Bl}_{p} M$ admits a cscK metric in the class $\pi^{*}[\omega]-\varepsilon^{2}[E]$,

(2) The pair $\left(\mathrm{Bl}_{p} M, \pi^{*}[\omega]-\varepsilon^{2}[E]\right)$ is K-stable with respect to smooth testconfigurations,

(3) There is a point $q$ in the $G^{c}$-orbit of $p$ such that $\mu(q)+\varepsilon \Delta \mu(q)=0$.

This extends our earlier result in [32, where $(M, \omega)$ was assumed to be a KählerEinstein manifold. A natural problem is to generalize this result to extremal metrics, and we will discuss the difficulty that arises in Section 3.2 .

The contents of the paper are as follows. In Section 2 we prove a finite dimensional perturbation result, which is sharper than the result we used in 32 . The heart of the paper is Section 3 where the main analytic gluing theorem, Theorem 12 is proved, and the proof of Theorem 1 is given at the end of Section 3.5. The main ingredient is a refined expansion of the solution to the equation introduced in [32. This is similar to other obstructed perturbation problems in the literature such as Pacard-Xu 22, but an important difference is that computing one more term in our case is enough to get a sharp existence result for $\csc$ metrics when $n>2$. The reason for this is the algebro-geometric structure of the problem. We discuss this aspect in the final Section 4, where we extend [32, Theorem 5] to Kähler manifolds which are not necessarily algebraic, and we give the proof of Theorem 2 
Acknowledgements. I would like to thank Frank Pacard and Michael Singer for several useful discussions.

\section{Relative Stability}

In this section we will consider the action of a compact Lie group $G$ on a compact Kähler manifold $M$, which is Hamiltonian with respect to a Kähler form $\omega$. The action of $G$ extends to a holomorphic action of the complexification $G^{c}$. Let us write

$$
\mu: M \rightarrow \mathfrak{g}
$$

for the moment map, where we have identified $\mathfrak{g} \cong \mathfrak{g}^{*}$ using an invariant inner product. Since we want to work on Kähler manifolds which may not be algebraic, we will review the basic ideas from relative stability [31] in this setting. The usual GIT theory has been extended to this setting by several authors, for instance Mundet $\mathrm{i}$ Riera [20, and Teleman 33. Our definitions will not necessarily match with theirs since we just want to cover the bare minimum of the theory that we will use.

We will need to work with maximal compact subgroups of $G^{c}$ other than $G$. If $K \subset G^{c}$ is maximal compact, then $K=\operatorname{Ad}_{g}(G)$ for some $g \in G^{c}$. The metric $\left(g^{-1}\right)^{*} \omega$ is $K$-invariant, and a corresponding moment map is given by

$$
\mu_{K}(g \cdot p)=\operatorname{ad}_{g} \mu(p) .
$$

This way of assigning "compatible" moment maps for the actions of all maximal compact subgroups of $G^{c}$ is analogous to a linearization of the action in GIT, and it is called a "symplectization" of the action in [33.

Definition 3. (1) A point $p \in M$ is stable for the action of $G^{c}$ (and for our choice of symplectization), if the stabilizer $G_{p}^{c}$ is trivial, and there exists a point $q \in G^{c} \cdot p$ such that $\mu(q)=0$.

(2) A point $p \in M$ is semistable for the action of $G^{c}$ if there is a point in the orbit closure $q \in \overline{G^{c} \cdot p}$ such that $\mu(q)=0$.

(3) A point $p \in M$ is relatively stable for the action of $G^{c}$, if there exists a point $q \in G^{c} \cdot p$ such that $\mu(q) \in \mathfrak{g}_{q}$.

It follows from (3) that the definition is independent of the choice of maximal compact subgroup. The main observation in [31] (see also Kirwan [15]) is that relative stability of a point $p$ is equivalent to stability of $p$ for the action of a subgroup of $G^{c}$. The relevant subgroup can be defined for any complex torus $T^{c} \subset G^{c}$. For this, let $K \subset G^{c}$ be a maximal compact subgroup containing the compact torus $T$. Let us write

$$
\mathfrak{k}_{T^{\perp}}=\left\{\xi \in \mathfrak{k}: \operatorname{ad}_{\xi}(\eta)=0, \quad\langle\xi, \eta\rangle=0 \text { for all } \eta \in \mathfrak{t}\right\},
$$

and let $K_{T^{\perp}}$ be the corresponding subgroup of $K$ (this is a closed subgroup by [30, Lemma 1.3.2]). We then let $G_{T^{\perp}}^{c}$ be the complexification of $K_{T^{\perp}}$. We will need the following result.

Proposition 4. Suppose that $p \in M$ is relatively stable, and $T \subset G_{p}$ is a maximal torus, such that $T^{c} \subset G_{p}^{c}$ is also maximal. Then there exists a point $q \in G_{T^{\perp}}^{c} \cdot p$ such that $\mu(q) \in \mathfrak{g}_{q}$. 
Proof. Using (3), we can choose a maximal compact subgroup $K \subset G^{c}$ such that $\mu_{K}(p) \in \mathfrak{k}_{p}$. Let $T^{\prime} \subset K_{p}$ be a maximal torus. It follows then that $p$ has trivial stabilizer in the group $K_{T^{\prime}}$, and

$$
\operatorname{proj}_{\mathfrak{k}_{T^{\prime} \perp}} \mu_{K}(p)=0
$$

so from [32, Lemma 16] we find that the stabilizer of $p$ in $G_{T^{\prime \perp}}^{c}$ is trivial. This implies that $T^{\prime c}$ is a maximal torus in $G_{p}^{c}$. We can therefore conjugate $T^{\prime c}$ into $T^{c}$ using an element of $G_{p}^{c}$, and by taking the corresponding conjugate of the maximal compact $K$, we can assume that $T^{\prime}=T$.

From (44) we then have that $p$ is stable for the action of $G_{T^{\perp}}^{c}$, and in particular using the maximal compact subgroup $G_{T^{\perp}}$ we find that there is a point $q \in G_{T^{\perp}}^{c} \cdot p$ such that

$$
\operatorname{proj}_{\mathfrak{g}_{T \perp}} \mu(q)=0 .
$$

Since elements in $G_{T^{\perp}}^{c}$ commute with $T$, we have that $T \subset G_{q}$. Since $\mu(q)$ is $G_{q}$ invariant, we have that $\mu(q)$ commutes with $\mathfrak{t}$. It then follows from (5) that $\mu(q) \in \mathfrak{t}$, and in particular $\mu(q) \in \mathfrak{g}_{q}$. This is what we set out to prove.

We will also need a Kähler version of the Hilbert-Mumford criterion, developed in [20] and [33. For any $\xi \in \mathfrak{g}$ and $p \in M$ we define the weight

$$
W(p, \xi)=\lim _{t \rightarrow-\infty}\left\langle\mu\left(e^{i t \xi} \cdot p\right), \xi\right\rangle .
$$

We then have the following result from Teleman 33 .

Proposition 5. Suppose that the stabilizer $G_{p}^{c}$ is trivial.

(1) The point $p \in M$ is stable, i.e. there is $q \in G^{c} \cdot p$ with $\mu(q)=0$, if and only if there is a constant $\delta>0$, such that $W(p, \xi)>\delta$ for all $\xi$ with $\|\xi\|=1$.

(2) The point $p \in M$ is semistable, i.e. there is $q \in \overline{G^{c} \cdot p}$ with $\mu(q)=0$, if and only if $W(p, \xi) \geqslant 0$ for all $\xi \in \mathfrak{g}$.

Note that the strict lower bound $\delta>0$ in (1) is obtained because $W(p, \xi)$ as a function on the unit sphere in $\mathfrak{g}$ is lower semicontinuous, being the supremum of a family of continuous functions.

2.1. A perturbation problem. We will now assume that we have two Kähler forms $\omega, \eta$ on $M$, and the action of $G$ is assumed to be Hamiltonian with respect to both Kähler forms. We have equivariant moment maps

$$
\mu, \nu: M \rightarrow \mathfrak{g} .
$$

We will therefore have to specify which moment map (or symplectization) we use when we speak of stable or semistable points. In our application we will have $\nu=\mu+\delta_{0} \Delta \mu$ for small $\delta_{0}$, corresponding to the Kähler form $\omega-\delta_{0} \rho$. We will again assume that $p \in M$ has trivial stabilizer. Our goal is the following result.

Theorem 6. Let $p$ have trivial stabilizer. Suppose that $\mu_{\varepsilon}: M \rightarrow \mathfrak{g}$ is a family of continuous functions such that

$$
\mu_{\varepsilon}=\mu+\varepsilon \nu+O\left(\varepsilon^{\kappa}\right),
$$

for some $\kappa>1$. Suppose that for all sufficiently small $\varepsilon>0$ we can find $q \in G^{c} \cdot p$ such that $\mu(q)+\varepsilon \nu(q)=0$. Then for sufficiently small $\varepsilon>0$ we can find $q \in G^{c} \cdot p$ such that $\mu_{\varepsilon}(q)=0$. 
Proof. The assumption implies that for each small $\varepsilon>0$ we have a point $q_{\varepsilon} \in G^{c} \cdot p$ such that

$$
\mu\left(q_{\varepsilon}\right)+\varepsilon \nu\left(q_{\varepsilon}\right)=0 .
$$

By compactness of $M$, we can assume up to choosing a subsequence, that $q_{\varepsilon} \rightarrow q_{0}$ as $\varepsilon \rightarrow 0$, for some $q_{0} \in M$. It follows that

$$
\mu\left(q_{0}\right)=0 .
$$

Since $q_{0} \in \overline{G^{c} \cdot p}$, the point $p$ is semistable with respect to $\mu$. If $p$ were actually stable with respect to $\mu$, then we could apply Proposition 8 from 32 to find $q \in G^{c} \cdot p$ for sufficiently small $\varepsilon>0$ such that $\mu_{\varepsilon}(q)=0$. The difficulty now is that the point $q_{0}$ may be on the boundary of the $G^{c}$-orbit of $p$.

By the assumption, we can choose a small $\delta>0$ such that $p$ is stable with respect to $\mu+\delta \nu$. For $\varepsilon \ll \delta$ we have

$$
\frac{\delta}{\delta-\varepsilon} \mu_{\varepsilon}=\mu+\frac{\varepsilon}{\delta-\varepsilon}(\mu+\delta \nu)+O\left(\varepsilon^{\kappa}\right),
$$

and so by replacing $\nu$ by $\mu+\delta \nu$, we can assume that $p$ is stable with respect to $\nu$.

Let us define the weights $W_{\mu}(p, \xi)$ and $W_{\nu}(p, \xi)$ as in (6). We then know from Proposition 5 that $W_{\mu}(p, \xi) \geqslant 0$ for all $\xi$, and there is a $c_{0}>0$ such that

$$
W_{\nu}(p, \xi)>c_{0} \text {, for all } \xi \text { with }\|\xi\|=1 \text {. }
$$

Fix an $\varepsilon>0$. By linearity we have $W_{\mu+\varepsilon \nu}(p, \xi)>0$ for all $\xi \neq 0$, and in fact

$$
W_{\mu+\varepsilon \nu}(p, \xi)>\varepsilon c_{0}, \text { for all } \xi \text { with }\|\xi\|=1 \text {. }
$$

Using the compactness of the unit sphere, it follows that there is a large radius $R>0$ such that

$$
\left\|\mu\left(e^{i \xi} \cdot p\right)+\varepsilon \nu\left(e^{i \xi} \cdot p\right)\right\|>\frac{1}{2} \varepsilon c_{0}, \text { for all } \xi \text { with }\|\xi\|=R .
$$

Since $p$ is stable with respect to $\mu+\varepsilon \nu$, it follows that there is a unique $\xi_{\varepsilon}$ such that

$$
\mu\left(e^{i \xi_{\varepsilon}} \cdot p\right)+\varepsilon \nu\left(e^{i \xi_{\varepsilon}} \cdot p\right)=0
$$

Consider the maps $F, F_{\varepsilon}: \mathfrak{g} \rightarrow \mathfrak{g}$ given by

$$
\begin{aligned}
F(\xi) & =\mu\left(e^{i \xi} \cdot p\right)+\varepsilon \nu\left(e^{i \xi} \cdot p\right) \\
F_{\varepsilon}(\xi) & =\mu_{\varepsilon}\left(e^{i \xi} \cdot p\right) .
\end{aligned}
$$

From (77) we know that $F$ induces a map

$$
F: \partial B(R) \rightarrow \mathfrak{g} \backslash\{0\},
$$

where $\partial B(R)$ is the $R$-sphere in $\mathfrak{g}$. By a homotopy argument we can see that the degree of this map is \pm 1 , since $F$ also induces a map

$$
F: \mathfrak{g} \backslash\left\{\xi_{\varepsilon}\right\} \rightarrow \mathfrak{g} \backslash\{0\},
$$

while at $\xi_{\varepsilon}$ the derivative of $F$ is an isomorphism. If $\varepsilon$ is chosen sufficiently small, then by our assumption

$$
\left|F_{\varepsilon}-F\right|<\frac{1}{4} \varepsilon c_{0},
$$

so $F_{\varepsilon}$ also defines a map with nonzero degree from $\partial B(R)$ to $\mathfrak{g} \backslash\{0\}$. But then there must be a $\xi \in \mathfrak{g}$ such that $F_{\varepsilon}(\xi)=0$. 
We also need the following, which is analogous to Proposition 12 in [32, but applies in the non-algebraic case as well.

Proposition 7. Assume again that the stabilizer of $p$ is trivial. There is a $\delta_{0}>0$, depending on $\mu, \nu$, such that the following are equivalent:

(1) For some $\delta \in\left(0, \delta_{0}\right)$ we can find $q \in G^{c} \cdot p$ such that $\mu(q)+\delta \nu(q)=0$.

(2) We have $W_{\mu}(p, \xi) \geqslant 0$ for all $\xi$, and $W_{\nu}(p, \xi)>0$ for all $\xi \neq 0$ for which $W_{\mu}(p, \xi)=0$.

(3) For all $\delta \in\left(0, \delta_{0}\right)$ we can find $q \in G^{c} \cdot p$ such that $\mu(q)+\delta \nu(q)=0$.

In particular whether or not we can find $q \in G^{c} \cdot p$ such that $\mu(q)+\delta \nu(q)=0$ is independent of $\delta \in\left(0, \delta_{0}\right)$.

Proof. Let us write $S_{1} \subset \mathfrak{g}$ for the unit sphere. To prove $(1) \Rightarrow(2)$, note that (1) implies that $W_{\mu+\delta \nu}(p, \xi)>0$ for all $\xi \neq 0$. Suppose first that $W_{\mu}(p, \xi)<0$ for some $\xi \in S_{1}$. This would imply that for all sufficiently small $\delta>0$ we have $W_{\mu+\delta \nu}(p, \xi)<0$, which is a contradiction if $\delta_{0}$ is chosen to be sufficiently small. So (1) implies that $W_{\mu}(p, \xi) \geqslant 0$ for all $\xi$. In addition if $\xi \neq 0$, but $W_{\mu}(p, \xi)=0$, then clearly we must have $W_{\nu}(p, \xi)>0$.

To prove $(2) \Rightarrow(3)$ note that the set of $\xi \in S_{1}$ for which $W_{\mu}(p, \xi)=0$ is a closed subset in $S_{1}$ by lower semicontinuity of $W$. It follows that there is a $c_{0}>0$ such that

$$
W_{\nu}(p, \xi)>c_{0} \text {, for all } \xi \in S_{1} \text { such that } W_{\mu}(p, \xi)=0 .
$$

The set of $\xi \in S_{1}$ for which $W_{\nu}(p, \xi)>c_{0}$ is an open set $U$, whose complement in $S_{1}$ is compact. Again by lower semicontinuity, $W_{\mu}(p, \xi)>c_{1}$ for some $c_{1}>0$ for all $\xi \in S_{1} \backslash U$. The boundedness of $W_{\nu}(p, \xi)$ for $\xi \in S_{1}$ then easily implies that for sufficiently small $\delta$ we have

$$
W_{\mu+\delta \nu}(p, \xi)>0 \text {, for all } \xi \neq 0 .
$$

The implication $(3) \Rightarrow(1)$ is immediate.

\section{THE GLUING THEOREM}

The goal of this section is to state and prove the main gluing theorem that we will use, namely Theorem 12 .

3.1. Preliminary discussion. We will use a technique very similar to that employed in our earlier work [32. The first step is to use cutoff functions to glue the extremal metric on $M$ to a model metric (the Burns-Simanca metric) on the blowup of $\mathbf{C}^{m}$ at the origin, scaled down by a factor of $\varepsilon^{2}$. The gluing is performed on a small annulus around the point $p$. This results in a metric $\omega_{\varepsilon}$ on $\mathrm{Bl}_{p} M$, whose scalar curvature is controlled, since the Burns-Simanca metric is scalar flat. The problem of perturbing $\omega_{\varepsilon}$ to an extremal metric can then be written as finding a zero of a map

$$
F: C^{\infty}\left(\mathrm{Bl}_{p} M\right) \times \mathfrak{h} \rightarrow C^{\infty}\left(\mathrm{Bl}_{p} M\right),
$$

where $\mathfrak{h}$ is the space of Hamiltonian holomorphic vector fields on $\mathrm{Bl}_{p} M$. For the exact form of $F$, see Section 3.5 and note that in practice we must work with various weighted Hölder spaces instead of $C^{\infty}\left(\mathrm{Bl}_{p} M\right)$. The main technical difficulty in constructing an extremal metric on the blowup $\mathrm{Bl}_{p} M$ is that in general the space $\mathfrak{h}$ has lower dimension than the space $\mathfrak{g}$ of Hamiltonian holomorphic vector fields on $M$. The way this manifests itself in the analysis is that it is more difficult to 
find a well-controlled right-inverse for the linearization of $F$ as $\varepsilon \rightarrow 0$. In [32] we overcome this problem by introducing a more general operator of the form

$$
\widetilde{F}: C^{\infty}\left(\mathrm{Bl}_{p} M\right) \times \mathfrak{g} \rightarrow C^{\infty}\left(\mathrm{Bl}_{p} M\right),
$$

such that if $f \in \mathfrak{h}$, then $\widetilde{F}(\varphi, f)=F(\varphi, f)$. So if we find a zero $\widetilde{F}(\varphi, f)=0$ with $f \in \mathfrak{h}$, then we have an extremal metric. A right-inverse is not hard to construct for the linearization of $\widetilde{F}$, so we find a solution $\left(\varphi_{p}, f_{p}\right)$. If we blow up at a different point $q$, then we obtain a different pair $\left(\varphi_{q}, f_{q}\right)$. The crucial point is to compute the leading terms in $f_{p}$ as $\varepsilon \rightarrow 0$. This can be done by finding better approximate solutions than $\omega_{\varepsilon}$. In [32] we found that the first non-trivial term is $\mu(p)$, using a technique similar to 3 to improve the approximate solution. A finite dimensional perturbation argument then shows that if the vector field $\mu(p)$ vanishes at $p$, then for small $\varepsilon$ there is a point $q \in G^{c} \cdot p$ such that $f_{q}$ vanishes at $q$. This gives us an extremal metric on $\mathrm{Bl}_{q} M$, but $\mathrm{Bl}_{q} M$ is biholomorphic to $\mathrm{Bl}_{p} M$.

To prove Theorem 1 we need to find more terms in the expansion of $f_{p}$ as $\varepsilon \rightarrow 0$, so we need to find better approximate solutions. This involves some extra terms which we have not considered in [32, see Section 3.10,

3.2. Possible generalizations. We will now briefly discuss the new difficulties that arise in trying to generalize our results.

3.2.1. The case $m=2$. The main issue with the case when $m=2$ is that we need to compute more terms in the expansion of $f$ in Theorem 12. This can most easily be seen in the formula for the Futaki invariant on a blowup in Corollary 36, since the term involving $\Delta h_{v}$ vanishes for $m=2$. The algebro-geometric formula has been computed to more terms in [32, and the problem is to construct sufficiently good approximate solutions to obtain a corresponding expansion of $f$. We believe that this should be possible, but it needs a deeper analysis of the linearized problem than what we have performed in the case $m>2$.

3.2.2. More blowup points. A more significant issue arises when we try to blow up more than one point, in contrast to previous works [1, 2, 3, 32, where the number of points made little difference. The new complication in Theorem 1 is that we need to perform a gluing construction at the points $q_{\delta}$ in the $G^{c}$-orbit of $p$, for which $\mu\left(q_{\delta}\right)+\delta \Delta \mu\left(q_{\delta}\right)$ vanishes at $q_{\delta}$, for arbitrarily small $\delta$. In the borderline case when $p$ is not relatively stable with respect to $\mu$, the corresponding points $q_{\delta}$ will approach the boundary of the $G^{c}$-orbit of $p$ as $\delta \rightarrow 0$. When there is only one blowup point, then this is not a problem, since the geometry of the manifolds $\mathrm{Bl}_{q_{\delta}} M$ is controlled as $\delta \rightarrow 0$. When we blow up an $n$-tuple $\left(p_{1}, \ldots, p_{n}\right)$, however, then as we approach the boundary of the orbit, some of the points may approach each other. In this case the geometry of the blowup is only controlled as long as the $n$-tuple stays away from the "large diagonal" in the $n$-fold product $M \times M \times \ldots \times M$, where at least two points coincide. This means that in order to use the same strategy as what we used in the proof of Theorem 1 we would need to obtain results analogous to Propositions 19 and 20, where the norm of the inverse will now depend on the distance of our $n$-tuple from the large diagonal in a suitable sense. Alternatively one may try to make contact with the results on constructing cscK or extremal metrics on iterated blowups, developed for Kähler surfaces in LeBrun-Singer [18, Rollin-Singer 23] and Tipler [35] for instance. 
3.2.3. Extremal metrics. When $(M, \omega)$ is $\operatorname{cscK}$, we were able to obtain a sharp existence result for cscK metrics on the blowups $\mathrm{Bl}_{p} M$. Many of the arguments can be adapted with little difficulty to extremal metrics, however we were not able to show that when the hypothesis of Theorem 1 fails, then the blowups $\left(\mathrm{Bl}_{p} M,\left[\omega_{\varepsilon}\right]\right)$ are relatively K-unstable for sufficiently small $\varepsilon$. The basic reason is that the inner product of vector fields lifted to a blowup is not the same as their inner product on $M$. The inner product enters in the definition of relative stability, and in order to obtain a sharp existence result, we would need a version of Theorem 10 where the inner product on $\mathfrak{g}$ is also perturbed in order to match with the inner product of lifted vector fields. In practice this means that we would need to compute more terms in the expansion of $f$, similarly to the $m=2$ case. An alternative approach would be to show directly that the map $p \mapsto f_{p}$ above can itself be thought of as a moment map for a suitable perturbed Kähler form on $M$ together with a perturbed inner product on $\mathfrak{g}$, without necessarily knowing the expansion explicitly.

3.3. Burns-Simanca metric. To obtain the first approximate solution $\omega_{\varepsilon}$, we want to glue the extremal metric $\omega$ on $M$ to a rescaling of a suitable model metric on $\mathrm{Bl}_{0} \mathbf{C}^{m}$, ie. on the blowup of $\mathbf{C}^{m}$ at the origin. This model metric is a scalar flat metric found by Burns (see LeBrun [16]) for $m=2$ and by Simanca [24] for $m \geqslant 3$. Away from the exceptional divisor it can be written in the form

$$
\eta=i \partial \bar{\partial}\left(\frac{1}{2}|w|^{2}+\psi(w)\right)
$$

where $w=\left(w_{1}, \ldots, w_{m}\right)$ are standard coordinates on $\mathbf{C}^{m}$. The function $\psi$ can be found by solving an ODE. We will need the following result from Gauduchon [14] about the asymptotics of $\psi$. Note that we use $i \partial \bar{\partial}$ as opposed to $d d^{c}$ which introduces a factor of 2 in our formula, and also our normalization of the volume of the exceptional divisor is different.

Lemma 8. If $m \geqslant 3$ then the Kähler potential for a suitable scaling of the BurnsSimanca metric

$$
\eta=i \partial \bar{\partial}\left(|w|^{2} / 2+\psi(w)\right)
$$

satisfies

$$
\psi(w)=-\frac{1}{2 \pi^{m-1}(m-2)}|w|^{4-2 m}+d_{1}|w|^{2-2 m}+d_{2}|w|^{6-4 m}+O\left(|w|^{4-4 m}\right),
$$

where $d_{1}>0$. The scaling of $\eta$ is such that the exceptional divisor has volume $\frac{1}{(m-1) !}$.

The important aspect of this result for us is the formula for the coefficient of the first term, and the sign of the second. Note that the scaling is chosen in such a way that if we construct $\omega_{\varepsilon}$ as in Section 3.4. then we end up with a metric in the class $\pi^{*}[\omega]-\varepsilon^{2}[E]$.

3.4. The metric $\omega_{\varepsilon}$ on $\mathrm{Bl}_{p} M$. Suppose as before that $\omega$ is an extremal Kähler metric on $M$. Let $X_{\mathbf{s}}$ be the Hamiltonian vector field corresponding to the scalar curvature $\mathbf{s}(\omega)$. Write $G$ for the Hamiltonian isometry group of $(M, \omega)$, so the Lie algebra $\mathfrak{g}$ of $G$ consists of holomorphic Killing fields with zeros.

Choose a point $p \in M$ where the vector field $X_{\mathbf{s}}$ vanishes, and let $T \subset G_{p}$ be a torus fixing $p$ whose Lie algebra contains $X_{\mathbf{s}}$. Let $H \subset G$ consist of the elements commuting with $T$ and let us write $\overline{\mathfrak{h}} \subset C^{\infty}(M)$ for the space of Hamiltonian 
functions of vector fields in the Lie algebra of $H$. Note that $\overline{\mathfrak{h}}$ contains the constants as well. Let us also write $\overline{\mathfrak{t}} \subset \overline{\mathfrak{h}}$ for the Hamiltonian functions corresponding to the subgroup $T \subset H$.

Given a small parameter $\varepsilon>0$, we will construct an approximate solution to our problem on $\mathrm{Bl}_{p} M$ in the Kähler class $\pi^{*}[\omega]-\varepsilon^{2}[E]$. For simplicity assume that the exponential map is defined on the unit ball in the tangent space $T_{p} M$ (if not, we can scale up the metric $\omega$ ). Choose local normal coordinates $z$ near $p$ such that the group $T$ acts by unitary transformations on the unit ball $B_{1}$ around $p$ (this is possible by linearizing the action, see Bochner-Martin [5] Theorem 8). In these coordinates we can write

$$
\omega=i \partial \bar{\partial}\left(|z|^{2} / 2+\varphi(z)\right)
$$

where $\varphi=O\left(|z|^{4}\right)$. At the same time the Burns-Simanca metric from Section 3.3 has the form

$$
\eta=i \partial \bar{\partial}\left(|w|^{2} / 2+\psi(w)\right)
$$

We glue $\varepsilon^{2} \eta$ to $\omega$ using a cutoff function in the annulus $B_{2 r_{\varepsilon}} \backslash B_{r_{\varepsilon}}$ in $M$, where $r_{\varepsilon}=\varepsilon^{\alpha}$ for some $\alpha<1$ to be chosen later. Under the gluing we will have $z=\varepsilon w$. To do this, let $\gamma: \mathbf{R} \rightarrow[0,1]$ be smooth such that $\gamma(x)=0$ for $x<1$ and $\gamma(x)=1$ for $x>2$. Define

$$
\gamma_{1}(r)=\gamma\left(r / r_{\varepsilon}\right)
$$

and write $\gamma_{2}=1-\gamma_{1}$. Then for small $\varepsilon$ we can define a Kähler metric $\omega_{\varepsilon}$ on $\mathrm{Bl}_{p} M$ which on the annulus $B_{1} \backslash B_{\varepsilon}$ is given by

$$
\omega_{\varepsilon}=i \partial \bar{\partial}\left(\frac{|z|^{2}}{2}+\gamma_{1}(|z|) \varphi(z)+\gamma_{2}(|z|) \varepsilon^{2} \psi\left(\varepsilon^{-1} z\right)\right) \text {. }
$$

Moreover outside $B_{2 r_{\varepsilon}}$ the metric $\omega_{\varepsilon}=\omega$ while inside the ball $B_{r_{\varepsilon}}$ we have $\omega_{\varepsilon}=\varepsilon^{2} \eta$. Note that the action of $T$ lifts to $\mathrm{Bl}_{p} M$ giving biholomorphisms, and that $\omega_{\varepsilon}$ is $T$ invariant.

In order to define the operator $\widetilde{F}$ from (9), we need to lift elements in $\overline{\mathfrak{h}}$ to $\mathrm{Bl}_{p} M$.

Definition 9. We define a linear map

$$
1: \overline{\mathfrak{h}} \rightarrow C^{\infty}\left(\mathrm{Bl}_{p} M\right)
$$

as follows. Decompose $\overline{\mathfrak{h}}$ into a direct sum $\overline{\mathfrak{h}}=\overline{\mathfrak{t}} \oplus \mathfrak{h}^{\prime}$ in such a way that each function in $\mathfrak{h}^{\prime}$ vanishes at $p$. Each $f \in \overline{\mathfrak{t}}$ corresponds to a holomorphic Hamiltonian vector field $X_{f}$ on $M$ vanishing at $p$. We then define $\mathbf{l}(f)$ to be the Hamiltonian function with respect to $\omega_{\varepsilon}$ of the holomorphic lift of $X_{f}$ to $\mathrm{Bl}_{p} M$, normalized so that $f=\mathbf{l}(f)$ outside $B_{1}$. For $f \in \mathfrak{h}^{\prime}$ we define $\mathbf{l}(f)=\gamma_{1} f$ near $p$ using the cutoff function $\gamma_{1}$ from above. We can then think of this $\mathrm{l}(f)$ as a function on $\mathrm{Bl}_{p} M$. Finally we can extend $\mathbf{l}$ to all of $\overline{\mathfrak{h}}$ by linearity.

Note that in contrast to 32 we are not assuming that $T \subset G_{p}$ is a maximal torus. This is necessary for technical reasons, namely we will want to be able to work with all $T$-invariant points at the same time. On the other hand it implies that even if $f \in \overline{\mathfrak{h}}$ corresponds to a holomorphic vector field vanishing at $p$, its lift $\mathbf{l}(f)$ will not give rise to a holomorphic vector field, unless $f \in \overline{\mathfrak{t}}$.

We will also need lifts corresponding to metrics other than $\omega_{\varepsilon}$. If $\Omega=\omega_{\varepsilon}+i \partial \bar{\partial} \Phi$ and $\Phi$ is $T$-invariant, then we define

$$
\mathbf{l}_{\Omega}(f)=\mathbf{l}(f)+\frac{1}{2} \nabla \Phi \cdot \nabla f .
$$


If $f \in \overline{\mathfrak{t}}$, then $\mathbf{l}_{\Omega}(f)$ is a Hamiltonian function for the vector field $X_{f}$, with respect to $\Omega$. In particular this has the following consequence.

Lemma 10. If $f \in \overline{\mathfrak{t}}$, then

$$
\int_{\mathrm{Bl}_{p} M} \mathbf{l}_{\Omega}(f) \Omega^{m}=\int_{\mathrm{Bl}_{p} M} \mathbf{l}(f) \omega_{\varepsilon}^{m} .
$$

Proof. This can be checked by using $\Omega_{t}=\omega_{\varepsilon}+t i \partial \bar{\partial} \Phi$, and differentiating with respect to $t$. Alternatively, in the algebraic case, note that the integral of the Hamiltonian function of a holomorphic vector field can be computed algebro-geometrically, so in particular it is independent of the metric.

3.5. The extremal metric equation. We will now write down what the operators $F$ and $\widetilde{F}$ in (8) and (9) look like. We have a torus $T \subset G$ fixing $p$, and we seek a $T$-invariant function $\varphi$ on $\mathrm{Bl}_{p} M$ such that $\omega_{\varepsilon}+i \partial \bar{\partial} \varphi$ is an extremal metric.

We need the following which can also be found in [3], [32].

Lemma 11. Suppose that $\varphi \in C^{\infty}\left(\mathrm{Bl}_{p} M\right)^{T}$ and $f \in \overline{\mathfrak{t}}$ such that

$$
\mathbf{s}\left(\omega_{\varepsilon}+i \partial \bar{\partial} \varphi\right)-\frac{1}{2} \nabla \mathbf{l}(f) \cdot \nabla \varphi=\mathbf{l}(f),
$$

where the gradient and inner product are computed with respect to the metric $\omega_{\varepsilon}$. Then $\omega_{\varepsilon}+i \partial \bar{\partial} \varphi$ is an extremal metric.

In order to solve Equation (11) as a perturbation problem, we will write it in the form

$$
\mathbf{s}\left(\omega_{\varepsilon}+i \partial \bar{\partial} \varphi\right)-\frac{1}{2} \nabla \mathbf{l}(\mathbf{s}+f) \cdot \nabla \varphi=\mathbf{l}(\mathbf{s}+f),
$$

where $\mathbf{s} \in \mathfrak{t}$ is the scalar curvature of the extremal metric $\omega$. The advantage of this is that we now seek $\varphi$ and $f$ which are small, or in other words, setting $\varphi=0$ and $f=0$ we get an approximate solution to the equation.

For any Kähler metric $\Omega$ let us define the operators $L_{\Omega}$ and $Q_{\Omega}$ by

$$
\mathbf{s}(\Omega+i \partial \bar{\partial} \varphi)=\mathbf{s}(\Omega)+L_{\Omega}(\varphi)+Q_{\Omega}(\varphi),
$$

where $L$ is the linearized operator. A simple computation shows that

$$
L_{\Omega}(\varphi)=-\Delta_{\Omega}^{2} \varphi-\operatorname{Ric}(\Omega)^{i \bar{j}} \varphi_{i \bar{j}},
$$

and analysing this operator will be crucial later on. Note that we are using the complex Laplacian here which is half of the usual Riemannian one. The linear operator appearing in the linearization of Equation (12) is then

$$
(\varphi, f) \mapsto L_{\Omega_{\varepsilon}}(\varphi)-\frac{1}{2} \nabla \mathbf{l}(\mathbf{s}) \cdot \nabla \varphi-\mathbf{l}(f),
$$

which is closely related to the Lichnerowicz operator that we will discuss in Section 3.6.

We can now state the main gluing result that we will prove, which corresponds to finding a zero of the operator $\widetilde{F}$ in (9). The proof of this theorem will appear at the end of Section 3.12 . 
Theorem 12. Fix a torus $T \subset G$ such that $\mathbf{s} \in \overline{\mathfrak{t}}$. There are constants $\varepsilon_{0}>0$ and $\kappa>2 m$ with the following property. Suppose that $p \in M$ is a fixed point of $T$. For every $\varepsilon \in\left(0, \varepsilon_{0}\right)$ we can find $u \in C^{4, \alpha}\left(\mathrm{Bl}_{p} M\right)^{T}$ and $f \in \overline{\mathfrak{h}}$ satisfying the equation

$$
\mathbf{s}\left(\omega_{\varepsilon}+i \partial \bar{\partial} u\right)=\mathbf{l}_{\omega_{\varepsilon}+i \partial \bar{\partial} u}(f)=\mathbf{l}(f)+\frac{1}{2} \nabla \mathbf{l}(f) \cdot \nabla u .
$$

In addition the element $f \in \overline{\mathfrak{h}}$ has an expansion

$$
f=\mathbf{s}+C-\varepsilon^{2 m-2}\left(c_{1}-\frac{\varepsilon^{2}}{m !} \mathbf{s}(p)\right) \mu(p)-\varepsilon^{2 m} c_{2} \Delta \mu(p)+O\left(\varepsilon^{\kappa}\right),
$$

for some $\kappa>2 m$, where $C$ is a constant depending on $\varepsilon$, and $c_{1}, c_{2}>0$.

Assuming this result, we can prove Theorem 1.

Proof of Theorem 1. By replacing $p$ with a different point in its $G^{c}$-orbit, we can assume that the stabilizer $G_{p}$ is a maximal compact subgroup of the complex stabilizer $G_{p}^{c}$. Since the scalar curvature $\mathbf{s}$ is $G$-invariant, the new point will also be a critical point of $\nabla \mathbf{s}$.

Let $T \subset G_{p}$ be a maximal torus. Then $T^{c} \subset G_{p}^{c}$ is also a maximal torus, and writing $\mathfrak{t}$ for the Lie algebra, we have $\mathbf{s} \in \mathfrak{t}$. Let $H \subset G$ consist of the elements of $G$ commuting with $T$. As above, we write $\overline{\mathfrak{h}}$ for functions on $M$ whose Hamiltonian vector fields are in $\mathfrak{h}$. We apply Theorem 12 to the set $M^{T}$ of $T$ invariant points in $M$. For every $q \in M^{T}$ and $\varepsilon \in\left(0, \varepsilon_{0}\right)$ we obtain a $T$-invariant function $u_{q, \varepsilon} \in C^{4, \alpha}\left(\mathrm{Bl}_{q} M\right)$ and $f_{q, \varepsilon} \in \overline{\mathfrak{h}}$ such that if $f_{q, \varepsilon} \in \overline{\mathfrak{t}}$, then we have an extremal metric on $\mathrm{Bl}_{q} M$. From Theorem 12 we know that

$$
f_{q, \varepsilon}=\mathbf{s}+C-\varepsilon^{2 m-2}\left(c_{1}-\frac{\varepsilon^{2}}{m !} \mathbf{s}(q)\right) \mu(q)-\varepsilon^{2 m} c_{2} \Delta \mu(q)+O\left(\varepsilon^{\kappa}\right),
$$

where $\kappa>2 m, C$ is a constant depending on $\varepsilon$, and $c_{1}, c_{2}>0$. Let us write

$$
\begin{aligned}
\mu_{\varepsilon}(q) & =-\varepsilon^{2-2 m}\left(c_{1}-\frac{\varepsilon^{2}}{m !} \mathbf{s}(q)\right)^{-1}\left(f_{q, \varepsilon}-\mathbf{s}-C\right) \\
& =\mu(q)+\varepsilon^{2} \frac{c_{2}}{c_{1}} \Delta \mu(q)+O\left(\varepsilon^{\kappa^{\prime}}\right),
\end{aligned}
$$

where $\kappa^{\prime}>2$. Then $f_{q, \varepsilon} \in \overline{\mathfrak{t}}$ if and only if $\mu_{\varepsilon}(q) \in \overline{\mathfrak{t}}$.

We will now apply Theorem [6 to the action of $G_{T^{\perp}}^{c}$ on $M$, where $G_{T^{\perp}} \subset H$ is the group introduced in Section 2, The corresponding moment maps $\mu_{T \perp}$ and $\Delta \mu_{T^{\perp}}$ are $\mu, \Delta \mu$ projected to $\mathfrak{g}_{T^{\perp}}$. From the assumption of Theorem 1 together with Proposition 4, we know that for some sufficiently small $\delta_{0}>0$ there is a point $q$ in the $G_{T^{\perp}}^{c}$-orbit of $p$ such that

$$
\mu(q)+\delta_{0} \Delta \mu(q) \in \mathfrak{g}_{q} .
$$

Moreover, since $T^{c}$ is a maximal torus in $G_{p}^{c}$, and $G_{T^{\perp}}$ commutes with $T$, we have $\mathfrak{g}_{q}=\mathfrak{t}$. It follows that

$$
\mu_{T^{\perp}}(q)+\delta_{0} \Delta \mu_{T^{\perp}}(q)=0 .
$$

Proposition 7 and Theorem 6 now imply that for all sufficiently small $\varepsilon>0$ we can find $q \in G_{T^{\perp}}^{c} \cdot p$ such that

$$
\operatorname{pr}_{\mathfrak{g}_{T} \perp} \mu_{\varepsilon}(q)=0 .
$$

By construction, $\mu_{\varepsilon}(q) \in \overline{\mathfrak{h}}$, i.e. $\mu_{\varepsilon}(q)$ commutes with $T$, so (16) implies that $\mu_{\varepsilon}(q) \in \overline{\mathfrak{t}}$. This implies that $f_{q, \varepsilon} \in \overline{\mathfrak{t}}$, and so we have obtained an extremal metric 
on $\mathrm{Bl}_{q} M$, in the class $\pi^{*}[\omega]-\varepsilon^{2}[E]$. Since $q \in G^{c} \cdot p$, the manifold $\mathrm{Bl}_{q} M$ is biholomorphic to $\mathrm{Bl}_{p} M$.

3.6. The Lichnerowicz operator. For any Kähler metric $\Omega$ on a manifold $X$ we have the operator

$$
\mathcal{D}_{\Omega}: C^{\infty}(X) \rightarrow \Omega^{0,1}\left(T^{1,0} X\right),
$$

given by $\mathcal{D}(\varphi)=\bar{\partial} \nabla^{1,0} \varphi$ where $\bar{\partial}$ is the natural $\bar{\partial}$-operator on the holomorphic tangent bundle. The Lichnerowicz operator is then the fourth order operator

$$
\mathcal{D}_{\Omega}^{*} \mathcal{D}_{\Omega}: C^{\infty}(X) \rightarrow C^{\infty}(X),
$$

whose significance is that the kernel consists of precisely those functions whose gradients are holomorphic vector fields. The relation to the operator in Equation (14) is that a computation (see eg. LeBrun-Simanca [17]) shows that

$$
\mathcal{D}_{\Omega}^{*} \mathcal{D}_{\Omega}(\varphi)=-L_{\Omega}(\varphi)+\frac{1}{2} \nabla \mathbf{s}(\Omega) \cdot \nabla \varphi .
$$

When comparing this to Equation (14), note that in general $\mathbf{s}\left(\Omega_{\varepsilon}\right)$ is not equal to $\mathbf{l}(\mathbf{s})$. The difference will be sufficiently small though.

3.7. The Lichnerowicz operator on weighted spaces. As in Arezzo-Pacard [1, 2, Arezzo-Pacard-Singer [3] and also [32, we need to study the invertibility of the linearized operator between suitable weighted Hölder spaces on the blowup $\mathrm{Bl}_{p} M$. First we need to understand the behaviour the Lichnerowicz operator on weighted spaces on the manifolds $M \backslash\{p\}$ and $\mathrm{Bl}_{p} \mathbf{C}^{m}$, and then obtain results about the blowup by "gluing" these spaces. This section is parallel to Section 5.1 in [32, but we need slightly different results.

Let us first consider $M_{p}=M \backslash\{p\}$ with the metric $\omega$. For functions $f: M_{p} \rightarrow \mathbf{R}$ we define the weighted norm

$$
\|f\|_{C_{\delta}^{k, \alpha}\left(M_{p}\right)}=\|f\|_{C_{\omega}^{k, \alpha}\left(M \backslash B_{1 / 2}\right)}+\sup _{r<1 / 2} r^{-\delta}\|f\|_{C_{r^{-2} \omega^{2}}^{k, \alpha}\left(B_{2 r} \backslash B_{r}\right)} .
$$

Here the subscripts $\omega$ and $r^{-2} \omega$ indicate the metrics used for computing the corresponding norm. The weighted space $C_{\delta}^{k, \alpha}\left(M_{p}\right)$ consists of functions on $M \backslash\{p\}$ which are locally in $C^{k, \alpha}$ and whose $\|\cdot\|_{C_{\delta}^{k, \alpha}}$ norm is finite.

We need the following result, which is Proposition 17 in [32. As before, we have a torus $T \subset G$ fixing the point $p$, and $H \subset G$ is the centralizer of $T$.

Proposition 13. If $\delta<0, \delta$ is not an integer, and $\alpha \in(0,1)$, then the operator

$$
\begin{aligned}
& C_{\delta}^{k, \alpha}\left(M_{p}\right)^{T} \times \overline{\mathfrak{t}} \rightarrow C_{\delta-4}^{k-4, \alpha}\left(M_{p}\right)^{T} \\
(\varphi, f) & \mapsto \mathcal{D}_{\omega}^{*} \mathcal{D}_{\omega} \varphi-f
\end{aligned}
$$

has a bounded right-inverse.

Let us turn now to the manifold $\mathrm{Bl}_{0} \mathbf{C}^{m}$ with the Burns-Simanca metric $\eta$. The relevant weighted Hölder norm is now given by

$$
\|f\|_{C_{\delta}^{k, \alpha}\left(\mathrm{Bl}_{0} \mathbf{C}^{m}\right)}=\|f\|_{C_{\eta}^{k, \alpha}\left(B_{2}\right)}+\sup _{r>1} r^{-\delta}\|f\|_{C_{r-2 \eta}^{k, \alpha}\left(B_{2 r} \backslash B_{r}\right)} .
$$

Here we abused notation slightly by writing $B_{r} \subset B l_{0} \mathbf{C}^{m}$ for the set where $|z|<r$ (ie. the pullback of the $r$-ball in $\mathbf{C}^{m}$ under the blowdown map).

The following is Proposition 18 from 32 . 
Proposition 14. If $\delta>4-2 m$ the operator

$$
\begin{aligned}
C_{\delta}^{k, \alpha}\left(\mathrm{Bl}_{0} \mathbf{C}^{m}\right) & \rightarrow C_{\delta-4}^{k-4, \alpha}\left(\mathrm{Bl}_{0} \mathbf{C}^{m}\right) \\
\varphi & \mapsto \mathcal{D}_{\eta}^{*} \mathcal{D}_{\eta} \varphi
\end{aligned}
$$

has a bounded right inverse.

If $\delta \in(3-2 m, 4-2 m)$, let $\chi$ be a compactly supported function on $\mathrm{Bl}_{0} \mathbf{C}^{m}$ with non-zero integral. The operator

$$
\begin{aligned}
C_{\delta}^{k, \alpha}\left(\mathrm{Bl}_{0} \mathbf{C}^{m}\right) \times \mathbf{R} & \rightarrow C_{\delta-4}^{k-4, \alpha}\left(\mathrm{Bl}_{0} \mathbf{C}^{m}\right), \\
(\varphi, t) & \mapsto \mathcal{D}_{\eta}^{*} \mathcal{D}_{\eta}(\varphi)+t \chi
\end{aligned}
$$

has a bounded right inverse.

3.8. Weighted spaces on $\mathrm{Bl}_{p} M$. We will need to do analysis on the blown-up manifold $\mathrm{Bl}_{p} M$ endowed with the approximately extremal metric $\omega_{\varepsilon}$. For this we define the following weighted spaces, which are simply glued versions of the above weighted spaces on $M \backslash\{p\}$ and $\mathrm{Bl}_{p} \mathbf{C}^{m}$.

We define the weighted Hölder norms $C_{\delta}^{k, \alpha}$ by

$$
\|f\|_{C_{\delta}^{k, \alpha}}=\|f\|_{C_{\omega}^{k, \alpha}\left(M \backslash B_{1}\right)}+\sup _{\varepsilon \leqslant r \leqslant 1 / 2} r^{-\delta}\|f\|_{C_{r^{-2} \omega_{\varepsilon}}^{k, \alpha}\left(B_{2 r} \backslash B_{r}\right)}+\varepsilon^{-\delta}\|f\|_{C_{\eta}^{k, \alpha}\left(B_{\varepsilon}\right)} .
$$

The subscripts indicate the metrics used to compute the relevant norm. This is a glued version of the two spaces defined in the previous section in the following sense. If $f \in C^{k, \alpha}\left(\mathrm{Bl}_{p} M\right)$ and we think of $\mathrm{Bl}_{p} M$ as a gluing of $M \backslash\{p\}$ and $\mathrm{Bl}_{0} \mathbf{C}^{m}$ then $\gamma_{1} f$ and $\gamma_{2} f$ can naturally be thought of as functions on $M \backslash\{p\}$ and $\mathrm{Bl}_{0} \mathbf{C}^{m}$ respectively. Then the norm $\|f\|_{C_{\delta}^{k, \alpha}\left(\mathrm{Bl}_{p} M\right)}$ is comparable to

$$
\left\|\gamma_{1} f\right\|_{C_{\delta}^{k, \alpha}\left(M_{p}\right)}+\varepsilon^{-\delta}\left\|\gamma_{2} f\right\|_{C_{\delta}^{k, \alpha}\left(\mathrm{Bl}_{0} \mathbf{C}^{m}, \eta\right)} .
$$

Another way to think about the norm is that if $\|f\|_{C_{\delta}^{k, \alpha}} \leqslant c$ then $f$ is in $C^{k, \alpha}\left(\mathrm{Bl}_{p} M\right)$ and also for $i \leqslant k$ we have

$$
\begin{gathered}
\left|\nabla^{i} f\right| \leqslant c \text { for } r \geqslant 1 \\
\left|\nabla^{i} f\right| \leqslant c r^{\delta-i} \text { for } \varepsilon \leqslant r \leqslant 1 \\
\left|\nabla^{i} f\right| \leqslant c \varepsilon^{\delta-i} \text { for } r \leqslant \varepsilon .
\end{gathered}
$$

The norms here are computed with respect to the metric $\omega_{\varepsilon}$, and note that on $B_{\varepsilon}$ we have $\omega_{\varepsilon}=\varepsilon^{2} \eta$. We will often use the following to compare the different weighted norms:

$$
\|f\|_{C_{\delta}^{k, \alpha}} \leqslant\left\{\begin{array}{l}
\|f\|_{C_{\delta^{\prime}}^{k, \alpha},} \text { if } \delta^{\prime}>\delta, \\
\varepsilon^{\delta^{\prime}-\delta}\|f\|_{\delta_{\delta^{\prime}}^{k, \alpha}}, \text { if } \delta^{\prime}<\delta .
\end{array}\right.
$$

Sometimes we will restrict the norm to subsets such as $C_{\delta}^{k, \alpha}\left(M \backslash B_{r_{\varepsilon}}\right)$ and $C_{\delta}^{k, \alpha}\left(B_{2 r_{\varepsilon}}\right)$. A crucial property of these weighted norms is that

$$
\left\|\gamma_{i}\right\|_{C_{0}^{4, \alpha}} \leqslant c
$$

for some constant $c$ independent of $\varepsilon$, where $\gamma_{i}$ are the cutoff functions from Section 3.4 .

In addition we need the following lemma about lifting elements of $\overline{\mathfrak{h}} \subset C^{\infty}(M)$ to $C^{\infty}\left(\mathrm{Bl}_{p} M\right)$ according to Definition 9 . 
Lemma 15. For any $f \in \overline{\mathfrak{h}}$ its lifting satisfies

$$
\begin{array}{r}
\|\mathbf{l}(f)\|_{C_{0}^{1, \alpha}} \leqslant c|f|, \\
\|\nabla \mathbf{l}(f)\|_{C_{0}^{1, \alpha}} \leqslant c|f|,
\end{array}
$$

for some constant $c$ independent of $\varepsilon$. Here $|\cdot|$ is any fixed norm on $\overline{\mathfrak{h}}$.

Proof. Recall that we defined the lifting using a decomposition $\overline{\mathfrak{h}}=\overline{\mathfrak{t}} \oplus \mathfrak{h}^{\prime}$, where the functions in $\mathfrak{h}^{\prime}$ vanish at $p$. Suppose first that $f \in \mathfrak{h}^{\prime}$. Since $f$ vanishes at $p$, we have

$$
\|f\|_{C_{1}^{1, \alpha}\left(M_{p}\right)} \leqslant c|f|
$$

where $c$ is independent of $f$. It follows from the multiplication properties of weighted spaces and (18) that

$$
\|\mathbf{l}(f)\|_{C_{1}^{1, \alpha}} \leqslant c|f|
$$

from which the required inequalities follow.

Now suppose that $f \in \overline{\mathfrak{t}}$, and write $X_{f}$ for the holomorphic vector field on $M$ corresponding to $f$. The result is clearly true for constants, so we can assume that $f$ vanishes at $p$. On the ball $B_{r_{\varepsilon}} \subset M$, the action of $X_{f}$ is given by unitary transformations, generated by a matrix $A$, say. Outside $B_{r_{\varepsilon}}$ the vector field is unchanged and the metrics $\omega$ and $\omega_{\varepsilon}$ are uniformly equivalent. Inside $B_{r_{\varepsilon}}$ the metric $\omega_{\varepsilon}$ is uniformly equivalent to $\varepsilon^{2} \eta$. It is more convenient to work with $\eta$, since that is a fixed metric, and we can then scale back depending on $\varepsilon$. Let $f_{\eta}$ be the Hamiltonian function of $X_{f}$ with respect to $\eta$, so $f=\varepsilon^{2} f_{\eta}$. In terms of $\eta$ we are working on the ball $B_{R_{\varepsilon}}$, and outside $B_{1}$ the Hamiltonian $f_{\eta}$ is given by a quadratic function depending on $A$. It follows that we have pointwise bounds

$$
\left|\nabla^{i} f_{\eta}(x)\right|_{\eta} \leqslant C_{i} r(x)^{2-i}|A|,
$$

where $r(x)=1$ inside $B_{1}$, and $r(x)$ is the distance from the exceptional divisor outside $B_{1}$. We can choose the norm $|A|$ to coincide with the norm $|f|$ chosen on the finite dimensional vector space $\overline{\mathfrak{h}}$. Rescaling this inequality, together with what we already know outside $B_{r_{\varepsilon}}$, we get $\|\mathbf{l}(f)\|_{C_{2}^{k, \alpha}} \leqslant C|f|$, which implies the results that we want.

3.9. The linearized operator on $\mathrm{Bl}_{p} M$. We now begin studying the linearized operator on $\mathrm{Bl}_{p} M$, in terms of the weighted spaces introduced in the previous section. The constants that appear below will be independent of $\varepsilon$ unless the dependence is made explicit.

Recall that for any metric $\Omega$ we write

$$
L_{\Omega}(\varphi)=-\Delta_{\Omega}^{2} \varphi-\operatorname{Ric}(\Omega)^{i \bar{j}} \varphi_{i \bar{j}} .
$$

We want to first study how this varies as we change the metric. For this we have the following, which is Proposition 20 from 32.

Proposition 16. Suppose that $\delta<0$. There exist constants $c_{0}, C>0$ such that if $\|\varphi\|_{C_{2}^{4, \alpha}}<c_{0}$ then

$$
\left\|L_{\omega_{\varphi}}(f)-L_{\omega_{\varepsilon}}(f)\right\|_{C_{\delta-4}^{0, \alpha}} \leqslant C\|\varphi\|_{C_{2}^{4, \alpha}}\|f\|_{C_{\delta}^{4, \alpha}}
$$

where $\omega_{\varphi}=\omega_{\varepsilon}+i \partial \bar{\partial} \varphi$ 
One consequence is an estimate for the nonlinear operator $Q_{\omega_{\varepsilon}}$ in the formula

$$
\mathbf{s}\left(\omega_{\varepsilon}+i \partial \bar{\partial} \varphi\right)=\mathbf{s}\left(\omega_{\varepsilon}\right)+L_{\omega_{\varepsilon}}(\varphi)+Q_{\omega_{\varepsilon}}(\varphi) .
$$

The following is Lemma 21 in [32].

Lemma 17. Suppose that $\delta<0$. There exists a $c_{0}>0$ such that if

$$
\|\varphi\|_{C_{2}^{4, \alpha}},\|\psi\|_{C_{2}^{4, \alpha}} \leqslant c_{0}
$$

then

$$
\left\|Q_{\omega_{\varepsilon}}(\varphi)-Q_{\omega_{\varepsilon}}(\psi)\right\|_{C_{\delta-4}^{0, \alpha}} \leqslant C\left(\|\varphi\|_{C_{2}^{4, \alpha}}+\|\psi\|_{C_{2}^{4, \alpha}}\right)\|\varphi-\psi\|_{C_{\delta}^{4, \alpha}} .
$$

We will need one further result, which was not used in [32].

Lemma 18. Suppose that $\omega=\omega_{\varepsilon}+i \partial \bar{\partial} \varphi$, and

$$
\|\varphi\|_{C_{2}^{4, \alpha}},\|\psi\|_{C_{2}^{4, \alpha}} \leqslant c_{0}
$$

for some sufficiently small $c_{0}$. Then

$$
\left\|Q_{\omega}(\psi)-Q_{\omega_{\varepsilon}}(\psi)\right\|_{C_{\delta-4}^{0, \alpha}} \leqslant C\|\varphi\|_{C_{2}^{4, \alpha}}\|\psi\|_{C_{2}^{4, \alpha}}\|\psi\|_{C_{\delta}^{4, \alpha}} .
$$

Proof. Let us write $g$ for a metric, and $g+h$ for a small perturbation, thought of as matrices in local coordinates. We can write schematically

$$
\begin{aligned}
\mathbf{s}(g+h) & =(g+h)^{-1} \partial^{2} \log \operatorname{det}(g+h) \\
& =g^{-1}\left(I+g^{-1} h\right)^{-1} \partial^{2}\left(\log \operatorname{det} g+\log \operatorname{det}\left(I+g^{-1} h\right)\right),
\end{aligned}
$$

where $I$ is the identity matrix. Expanding in power series, we find that $Q$ is of the form

$$
Q_{g}(h)=\sum_{i=0}^{2} g^{-1}\left[\partial^{i}\left(g^{-1} h\right)^{2}\right] F_{i}\left(g^{-1} h\right),
$$

where the $F_{i}$ are power series. In order to estimate $Q_{g_{1}}(h)-Q_{g_{2}}(h)$ it is enough to consider a typical term, for instance

$$
g_{1}^{-1}\left[\partial^{2}\left(g_{1}^{-1} h\right)^{2}\right]\left(g_{1}^{-1} h\right)^{l}-g_{2}^{-1}\left[\partial^{2}\left(g_{2}^{-1} h\right)^{2}\right]\left(g_{2}^{-1} h\right)^{l},
$$

for some $l \geqslant 0$. In our situation $h=i \partial \bar{\partial} \psi$, and we have

$$
\begin{aligned}
\left\|g_{1}^{-1}-g_{2}^{-1}\right\|_{C_{0}^{2, \alpha}} & \leqslant C\|\varphi\|_{C_{2}^{4, \alpha}} \leqslant C c_{0}, \\
\left\|g_{j}^{-1} h\right\|_{C_{0}^{2, \alpha}} & \leqslant C\|\psi\|_{C_{2}^{4, \alpha}} \leqslant C c_{0}, \\
\left\|g_{j}^{-1} h\right\|_{C_{\delta-2}^{2, \alpha}} & \leqslant C\|\psi\|_{C_{\delta}^{4, \alpha}},
\end{aligned}
$$

for $j=1,2$. From this it is a straightforward calculation to check the estimate (20).

The heart of the matter is to understand the invertibility of the linearized operator of our problem on $\mathrm{Bl}_{p} M$. The following is Proposition 22 from 32 .

Proposition 19. For sufficiently small $\varepsilon$ and $\delta \in(4-2 m, 0)$ the operator

$$
\begin{gathered}
G_{1}:\left(C_{\delta}^{4, \alpha}\right)^{T} \times \overline{\mathfrak{h}} \rightarrow\left(C_{\delta-4}^{0, \alpha}\right)^{T} \\
(\varphi, f) \mapsto L_{\omega_{\varepsilon}}(\varphi)-\frac{1}{2} \nabla \mathbf{s}\left(\omega_{\varepsilon}\right) \cdot \nabla \varphi-\mathbf{l}(f)
\end{gathered}
$$

has a right inverse $P_{1}$, with the operator norm $\left\|P_{1}\right\|<C$ for some constant $C$ independent of $\varepsilon$. 
We will need a slight variation of this result as well, dealing with weights in the range $(3-2 m, 4-2 m)$. One can easily obtain a result for $\delta \in(3-2 m, 4-2 m)$ from the preivous proposition, but we will only have a bound of the form $C \varepsilon^{\delta-(4-2 m)}$ for the inverse. It turns out that if we restrict the range to functions with zero mean, we can obtain an inverse with norm bounded independent of $\varepsilon$.

Proposition 20. Let us write $\left(C_{\delta-4}^{0, \alpha}\right)_{0}^{T}$ for the elements in $\left(C_{\delta-4}^{0, \alpha}\right)^{T}$ which have zero mean on $\mathrm{Bl}_{p} M$, and $\overline{\mathfrak{h}}_{0}$ for the elements $f \in \overline{\mathfrak{h}}$ such that $\mathrm{l}(f)$ has zero mean on $\mathrm{Bl}_{p} M$ with respect to $\omega_{\varepsilon}$. For sufficiently small $\varepsilon$, and $\delta \in(3-2 m, 4-2 m)$, the operator

$$
\begin{gathered}
G_{2}:\left(C_{\delta}^{4, \alpha}\right)^{T} \times \overline{\mathfrak{h}}_{0} \rightarrow\left(C_{\delta-4}^{0, \alpha}\right)_{0}^{T} \\
(\varphi, f) \mapsto L_{\omega_{\varepsilon}}(\varphi)-\frac{1}{2} \nabla \mathbf{s}\left(\omega_{\varepsilon}\right) \cdot \nabla \varphi-\mathbf{l}(f)
\end{gathered}
$$

has a right inverse $P_{2}$, with the operator norm $\left\|P_{2}\right\|<C$ for some constant $C$ independent of $\varepsilon$.

Proof. The proof is very similar to the proof of Proposition 22 in [32], for the $m=2$ case. The idea is to first work with the operator

$$
\begin{aligned}
G_{0}:\left(C_{\delta}^{4, \alpha}\right)^{T} \times \overline{\mathfrak{h}}_{0} \times \mathbf{R} & \rightarrow\left(C_{\delta-4}^{0, \alpha}\right)^{T} \\
(\varphi, f, t) & \mapsto \mathcal{D}_{\omega_{\varepsilon}}^{*} \mathcal{D}_{\omega_{\varepsilon}}-\mathbf{l}(f)+t \chi,
\end{aligned}
$$

where $\chi$ is the function from Proposition 14 and

$$
\mathcal{D}_{\omega_{\varepsilon}}^{*} \mathcal{D}_{\omega_{\varepsilon}}=-L_{\omega_{\varepsilon}}(\varphi)+\frac{1}{2} \nabla \mathbf{s}\left(\omega_{\varepsilon}\right) \cdot \nabla \varphi .
$$

One can then use the inverses in Propositions 13 and 14 to construct an approximate right inverse for $G_{0}$, which in turn can be used to show that $G_{0}$ has a bounded right inverse. If $\psi \in\left(C_{\delta-4}^{0, \alpha}\right)_{0}^{T}$, then we can use this to find $\varphi \in\left(C_{\delta}^{4, \alpha}\right)^{T}, f \in \overline{\mathfrak{h}}_{0}, t \in \mathbf{R}$ such that

$$
\mathcal{D}_{\omega_{\varepsilon}}^{*} \mathcal{D}_{\omega_{\varepsilon}}(\varphi)-\mathbf{l}(f)+t \chi=\psi
$$

Integrating this over $\mathrm{Bl}_{p} M$ we find that $t=0$. This shows that we have constructed an inverse for $G_{1}$.

Remark 21. We will need analogous results for operators corresponding to a perturbation $\Omega=\omega_{\varepsilon}+i \partial \bar{\partial} \Phi$. We have

$$
\left\|\left(\mathcal{D}_{\omega_{\varepsilon}}^{*} \mathcal{D}_{\omega_{\varepsilon}}-\mathcal{D}_{\Omega}^{*} \mathcal{D}_{\Omega}\right) \varphi\right\|_{C_{\delta-4}^{0, \alpha}} \leqslant C\|\Phi\|_{C_{2}^{4, \alpha}}\|\varphi\|_{C_{\delta}^{4, \alpha}}
$$

and

$$
\left\|\mathbf{l}(f)-\mathbf{l}_{\Omega}(f)\right\|_{C_{\delta-4}^{0, \alpha}} \leqslant C\|\nabla \mathbf{l}(f) \cdot \nabla \Phi\|_{C_{\delta-4}^{0, \alpha}} \leqslant C|f|\|\Phi\|_{C_{\delta-3}^{4, \alpha}} \leqslant C|f|\|\Phi\|_{C_{2}^{4, \alpha}},
$$

if $\delta-3<2$. So as long as $\|\Phi\|_{C_{2}^{4, \alpha}}$ is sufficiently small, we can deduce the invertibility of the operators corresponding to $\Omega$ from the invertibility of those corresponding to $\omega_{\varepsilon}$. Note also that

$$
\begin{aligned}
\left|\int_{\mathrm{Bl}_{p} M} \mathbf{l}_{\Omega}(f) \Omega^{m}-\int_{\mathrm{Bl}_{p} M} \mathbf{l}(f) \omega_{\varepsilon}^{m}\right| & \leqslant \int_{\mathrm{Bl}_{p} M} \frac{1}{2}|\nabla \mathbf{l}(f) \cdot \nabla \Phi| \Omega^{m} \\
& +\left|\int_{\mathrm{Bl}_{p} M} \mathbf{l}(f)\left(\Omega^{m}-\omega_{\varepsilon}^{m}\right)\right| \\
& \leqslant C|f|\|\Phi\|_{C_{2}^{4, \alpha}}
\end{aligned}
$$


so if $f \in \overline{\mathfrak{h}_{0}}$, then we can adjust $f$ while preserving its norm up to a factor, to ensure that $\mathbf{l}_{\Omega}(f)$ has zero integral with respect to $\Omega$, as long as $\|\Phi\|_{C_{2}^{4, \alpha}}$ is sufficiently small. It follows that both propositions can be applied to small perturbations of $\omega_{\varepsilon}$.

3.10. The approximate solution $\Omega_{1}$. We will now work on obtaining a metric $\Omega_{1}$ on $\mathrm{Bl}_{p} M$, which is closer to being extremal than our previous candidate $\omega_{\varepsilon}$. In the next section we will use this to find an even better approximate solution $\Omega_{2}$, at which point we will be able to use the contraction mapping theorem to obtain a solution of our equation.

There are 3 regions in $\mathrm{Bl}_{p} M$ which we need to think about differently, namely the region $\mathrm{Bl}_{p} M \backslash B_{2 r_{\varepsilon}}$, the annular region $B_{2 r_{\varepsilon}} \backslash B_{r_{\varepsilon}}$ on which our cutoff fuctions $\gamma_{1}$ and $\gamma_{2}$ live, and $B_{r_{\varepsilon}}$. Here $r_{\varepsilon}=\varepsilon^{\alpha}$, and from now on we will work with

$$
\alpha=\frac{2 m-1}{2 m+1} .
$$

Like in Section 3.4, let us write the metric $\omega$ in coordinates near the point $p$. We will need a more precise expansion than before, so we write

$$
\omega=i \partial \bar{\partial}\left(\frac{|z|^{2}}{2}+A_{4}(z)+A_{5}(z)+\varphi_{6}(z)\right)
$$

where $A_{4}(z), A_{5}(z)$ are quartic and quintic in $z$ respectively, and $\varphi_{6} \in C_{6}^{k, \alpha}\left(M_{p}\right)$. Also let us write $\mathbf{s}=\mathbf{s}(\omega)$ for the scalar curvature of $\omega$.

Lemma 22. Suppose that $\nabla \mathbf{s}$ vanishes at $p$. Then we have

$$
\begin{aligned}
& \Delta_{0}^{2} A_{4}=-\mathbf{s}(p), \\
& \Delta_{0}^{2} A_{5}=0,
\end{aligned}
$$

where $\Delta_{0}$ is the Laplacian with respect to the Euclidean metric.

Proof. This follows from computing the scalar curvature of $\omega$ as a perturbation of the flat metric near $p$.

$$
\mathbf{s}(\omega)=-\Delta_{0}^{2}\left(A_{4}+A_{5}+\varphi_{6}\right)+Q_{0}\left(A_{4}+A_{5}+\varphi_{6}\right) .
$$

From Lemma 17 it follows that

$$
Q_{0}\left(A_{4}+A_{5}+\varphi_{6}\right) \in C_{2}^{k, \alpha}\left(B_{1} \backslash\{p\}\right),
$$

and so

$$
\mathbf{s}(\omega)+\Delta_{0}^{2}\left(A_{4}+A_{5}\right) \in C_{2}^{k, \alpha},
$$

near $p$. Since $\Delta_{0}^{2} A_{4}$ is a constant and $\Delta_{0}^{2} A_{5}$ is linear, the result follows.

As for the rescaled Burns-Simanca metric, after a change of coordinates we can write it as

$$
\begin{aligned}
\varepsilon^{2} \eta=i \partial \bar{\partial}\left(\frac{|z|^{2}}{2}\right. & +d_{0} \varepsilon^{2 m-2}|z|^{4-2 m}+d_{1} \varepsilon^{2 m}|z|^{2-2 m} \\
& \left.+d_{2} \varepsilon^{4 m-4}|z|^{6-4 m}+\varepsilon^{2} \psi_{4-4 m}\left(\varepsilon^{-1} z\right)\right),
\end{aligned}
$$

where $\psi_{4-4 m} \in C_{4-4 m}^{k, \alpha}\left(\mathrm{Bl}_{0} \mathbf{C}^{m}\right)$, and $d_{0}=-\frac{1}{2 \pi^{m-1}(m-2)}$.

These are the two metrics that we want to glue across the annular region $B_{2 r_{\varepsilon}} \backslash$ $B_{r_{\varepsilon}}$. In the construction of $\omega_{\varepsilon}$ we performed this gluing by multiplying all the terms except $|z|^{2} / 2$ by cutoff functions. In order to get a better approximate solution, 
we want to only multiply $\varphi_{6}$ and $\psi_{4-4 m}$ by cutoff functions. For this we need to modify $\varepsilon^{2} \eta$ so that it contains $A_{4}(z), A_{5}(z)$, and we need to modify $\omega$ by

$$
d_{0} \varepsilon^{2 m-2}|z|^{4-2 m}+d_{1} \varepsilon^{2 m}|z|^{2-2 m}+d_{2} \varepsilon^{4 m-4}|z|^{6-4 m} .
$$

Let us focus on $\omega$ first. For this we have the following.

Lemma 23. We can find T-invariant functions $G_{1}, G_{2}$ on $M \backslash\{p\}$ such that distributionally on $M$ we have

$$
\begin{aligned}
& \mathcal{D}_{\omega}^{*} \mathcal{D}_{\omega} G_{1}=f_{1}+\frac{4 \pi^{m}}{(m-3) !} \delta_{p} \\
& \mathcal{D}_{\omega}^{*} \mathcal{D}_{\omega} G_{2}=f_{2}-\frac{2 \pi^{m}}{(m-2) !} \Delta \delta_{p},
\end{aligned}
$$

for some $f_{1}, f_{2} \in \overline{\mathfrak{h}}$, and for any $\delta>0$ we have

$$
\begin{aligned}
& G_{1}-|z|^{4-2 m} \in C_{6-2 m-\delta}^{k, \alpha}\left(M_{p}\right), \\
& G_{2}-|z|^{2-2 m} \in C_{4-2 m-\delta}^{k, \alpha}\left(M_{p}\right) .
\end{aligned}
$$

In addition

where $V=\operatorname{Vol}(M)$.

$$
\begin{aligned}
& f_{1}=-\frac{4 \pi^{m}}{(m-3) !}\left(V^{-1}+\mu(p)\right), \\
& f_{2}=\frac{2 \pi^{m}}{(m-2) !} \Delta \mu(p)
\end{aligned}
$$

Proof. Let us define $\widetilde{G}_{1}$ using a cutoff function to be equal to zero on $M \backslash B_{1}$, and equal to $|z|^{4-2 m}$ on $B_{1 / 2}$. Comparing $\omega$ to the flat metric near $p$, we find that

$$
\mathcal{D}_{\omega}^{*} \mathcal{D}_{\omega} \widetilde{G}_{1} \in C_{2-2 m}^{k-4, \alpha}\left(M_{p}\right) \text {. }
$$

We can now use the inverse in Proposition 13. For small $\delta>0$ we have $6-2 m-\delta \in$ $(4-2 m, 0)$ (for $m>3$ we can let $\delta=0$ ), so we obtain a $\varphi \in C_{6-2 m-\delta}^{k, \alpha}$ and $f_{1} \in \overline{\mathfrak{h}}$ such that

$$
\mathcal{D}_{\omega}^{*} \mathcal{D}_{\omega}\left(\widetilde{G}_{1}-\varphi\right)=f_{1} \text {, on } M_{p},
$$

and so we can let $G_{1}=\widetilde{G}_{1}-\varphi$. The only contribution to the distributional part of $\mathcal{D}_{\omega}^{*} \mathcal{D}_{\omega} \widetilde{G}$ comes from $\Delta_{0}^{2}|z|^{4-2 m}$ in the flat metric, giving the result.

Similarly we define $\widetilde{G}_{2}$ using a cutoff function to equal $|z|^{2-2 m}$ on $B_{1 / 2}$ and to vanish outside $B_{1}$. Comparing with the flat metric again, we obtain

$$
\mathcal{D}_{\omega}^{*} \mathcal{D}_{\omega} \widetilde{G} \in C_{-2 m}^{k-4, \alpha}\left(M_{p}\right) .
$$

Once again we can find $\varphi \in C_{4-2 m-\delta}^{k, \alpha}$ for small $\delta>0$ (note that $4-2 m$ is an indicial root), and $f_{2} \in \overline{\mathfrak{h}}$ such that

$$
\mathcal{D}_{\omega}^{*} \mathcal{D}_{\omega}\left(\widetilde{G}_{2}-\varphi\right)=f_{2} \text {, on } M_{p} .
$$

In this case, there are several contributions to the distributional part at $p$, but apart from the leading contribution of $\Delta \delta_{p}$, the rest is a multiple of $\delta_{p}$. We can therefore find a constant $C$ such that $G_{2}=\widetilde{G}_{2}-\varphi+C G_{1}$ satisfies our requirements.

In order to find $f_{1}$, we take the $L^{2}$-product of (24) with any element $g \in \mathfrak{h}$, to obtain

$$
0=\left\langle f_{1}, g\right\rangle+\frac{4 \pi^{m}}{(m-3) !} g(p),
$$


since $\mathcal{D}_{\omega}^{*} \mathcal{D}_{\omega} g=0$. By definition $g(p)=\langle\mu(p), g\rangle$, and so it follows that the projection of $f_{1}$ onto $\mathfrak{h}$ must be

$$
\operatorname{pr}_{\mathfrak{h}} f_{1}=-\frac{4 \pi^{m}}{(m-3) !} \mu(p)
$$

To obtain $f_{1}$ from this, we just need to take the $L^{2}$-product of (24) with the function 1. We can obtain the formula for $f_{2}$ similarly.

Lemma 24. We can find a function $\Gamma$ on $M \backslash B_{r_{\varepsilon}}$ such that

$$
\mathcal{D}_{\omega}^{*} \mathcal{D}_{\omega} \Gamma=-h_{1} \text { on } M \backslash B_{1}
$$

for some $h_{1} \in \overline{\mathfrak{h}}$, and satisfying the following properties. On $B_{2 r_{\varepsilon}} \backslash B_{r_{\varepsilon}}$ the function $\Gamma$ has the form

$$
\Gamma=d_{0} \varepsilon^{2 m-2}|z|^{4-2 m}+d_{1} \varepsilon^{2 m}|z|^{2-2 m}+d_{2} \varepsilon^{4 m-4}|z|^{6-4 m}+\Gamma_{1},
$$

where

$$
\left\|\Gamma_{1}\right\|_{C_{3-2 m}^{k, \alpha}\left(B_{2 r_{\varepsilon}} \backslash B_{r_{\varepsilon}}\right)}=O\left(\varepsilon^{\kappa}\right),
$$

for some $\kappa>2 m$. On $M \backslash B_{2 r_{\varepsilon}}$ we have

$$
\left\|\mathbf{s}(\omega+i \partial \bar{\partial} \Gamma)-\frac{1}{2} \nabla \mathbf{s}(\omega) \cdot \nabla \Gamma-\mathbf{s}(\omega)-h_{1}\right\|_{C_{-1-2 m}^{0, \alpha}\left(M \backslash B_{2 \varepsilon}\right)}=O\left(\varepsilon^{\kappa}\right),
$$

for some $\kappa>2 m$. In addition

$$
\begin{aligned}
h_{1} & =-\frac{2 \pi \varepsilon^{2 m-2}}{(m-2) !}\left(V^{-1}+\mu(p)\right)+\frac{\varepsilon^{2 m} \mathbf{s}(p)}{m !}\left(V^{-1}+\mu(p)\right)-d_{1} \varepsilon^{2 m} \frac{2 \pi^{m}}{(m-2) !} \Delta \mu(p) \\
& =C-\varepsilon^{2 m-2}\left(c_{1}-\frac{\varepsilon^{2}}{m !} \mathbf{s}(p)\right) \mu(p)-\varepsilon^{2 m} c_{2} \Delta \mu(p),
\end{aligned}
$$

where $V=\operatorname{Vol}(M), C$ is a constant, and $c_{1}, c_{2}>0$.

Proof. Let us use a cutoff function to define $G_{3}$ to be zero outside $B_{1}$, and equal to $|z|^{6-4 m}$ in $B_{1 / 2}$. We let

$$
\Gamma=d_{0} \varepsilon^{2 m-2} G_{1}+d_{1} \varepsilon^{2 m} G_{2}-\varepsilon^{2 m} \frac{(m-3) ! \mathbf{s}(p)}{4 \pi^{m} m !} G_{1}+d_{2} \varepsilon^{4 m-4} G_{3},
$$

where $G_{1}$ and $G_{2}$ are defined in Lemma 23. Then (26) and (28) follow from the properties of $G_{1}, G_{2}$. On the annulus $B_{2 r_{\varepsilon}} \backslash B_{r_{\varepsilon}}$ we have

$$
\Gamma=d_{0} \varepsilon^{2 m-2}|z|^{4-2 m}+d_{1} \varepsilon^{2 m}|z|^{2-2 m}+d_{2} \varepsilon^{4 m-4}|z|^{6-4 m}+\Gamma_{1},
$$

where

$$
\Gamma_{1}=d_{0} \varepsilon^{2 m-2}\left(G_{1}-|z|^{4-2 m}\right)+d_{1} \varepsilon^{2 m}\left(G_{2}-|z|^{2-2 m}\right)+C \varepsilon^{2 m}|z|^{4-2 m},
$$

for some constant $C$. From (25) it follows that

$$
\left\|\Gamma_{1}\right\|_{C_{3-2 m}^{k, \alpha}\left(B_{2 r_{\varepsilon}} \backslash B_{r_{\varepsilon}}\right)}=O\left(\varepsilon^{\kappa}\right)
$$

for some $\kappa>2 m$. For instance

$$
\left\|G_{1}-|z|^{4-2 m}\right\|_{C_{3-2 m}^{k, \alpha}\left(B_{2 r_{\varepsilon}} \backslash B_{r_{\varepsilon}}\right)} \leqslant C r_{\varepsilon}^{3-\delta}\left\|G_{1}-|z|^{4-2 m}\right\|_{C_{6-2 m-\delta}^{k, \alpha}\left(B_{2 r_{\varepsilon}} \backslash B_{r_{\varepsilon}}\right)},
$$

and for sufficiently small $\delta>0$ we have

$$
\varepsilon^{2 m-2} r_{\varepsilon}^{3-\delta}=O\left(\varepsilon^{\kappa}\right)
$$


for some $\kappa>2 m$, by our choice of $r_{\varepsilon}$, since

$$
\frac{2 m-1}{2 m+1}>\frac{2}{3}
$$

for $m \geqslant 3$. The other terms are larger and are handled similarly.

For the scalar curvature of $\omega+i \partial \bar{\partial} \Gamma$ we have

$$
\mathbf{s}(\omega+i \partial \bar{\partial} \Gamma)-\frac{1}{2} \nabla \mathbf{s}(\omega) \cdot \nabla \Gamma-\mathbf{s}(\omega)-h_{1}=Q_{\omega}(\Gamma)-\mathcal{D}_{\omega}^{*} \mathcal{D}_{\omega} \Gamma-h_{1} .
$$

We will work on the 3 regions $M \backslash B_{1}, B_{1} \backslash B_{1 / 2}$ and $B_{1 / 2} \backslash B_{2 r_{\varepsilon}}$ separately.

On $M \backslash B_{1}$ we have $\mathcal{D}_{\omega}^{*} \mathcal{D}_{\omega} \Gamma+h_{1}=0$, and

$$
\left\|Q_{\omega}(\Gamma)\right\|_{C_{-1-2 m}^{0, \alpha}\left(M \backslash B_{1}\right)} \leqslant C\|\Gamma\|_{C_{2}^{4, \alpha}}\|\Gamma\|_{C_{3-2 m}^{4, \alpha}} .
$$

The weight is irrelevant outside $B_{1}$, so we have

$$
\left\|Q_{\omega}(\Gamma)\right\|_{C^{0, \alpha}\left(M \backslash B_{1}\right)} \leqslant C\left(\varepsilon^{2 m-2}\right)^{2}=O\left(\varepsilon^{\kappa}\right)
$$

for some $\kappa>2 m$, as long as $m>2$.

On $B_{1} \backslash B_{1 / 2}$ we can still ignore the weights, so we still have the same estimate for $Q_{\omega}(\Gamma)$, but now

$$
\mathcal{D}_{\omega}^{*} \mathcal{D}_{\omega} \Gamma+h_{1}=C \varepsilon^{4 m-4} \mathcal{D}_{\omega}^{*} \mathcal{D}_{\omega}\left(G_{3}\right) .
$$

It follows from this that

$$
\left\|\mathcal{D}_{\omega}^{*} \mathcal{D}_{\omega} \Gamma+h_{1}\right\|_{C^{0, \alpha}\left(B_{1} \backslash B_{1 / 2}\right)}=O\left(\varepsilon^{4 m-4}\right)=O\left(\varepsilon^{\kappa}\right)
$$

for $\kappa>2 m$, as long as $m>2$.

The most delicate estimate is on $B_{1 / 2} \backslash B_{r_{\varepsilon}}$. It is best to work on the annuli $A_{r}=B_{2 r} \backslash B_{r}$, for $r \in\left(r_{\varepsilon}, 1 / 4\right)$. We have

$$
\mathcal{D}_{\omega}^{*} \mathcal{D}_{\omega} \Gamma+h_{1}=d_{2} \varepsilon^{4 m-4} \mathcal{D}_{\omega}^{*} \mathcal{D}_{\omega}|z|^{6-4 m}
$$

SO

$\left\|\mathcal{D}_{\omega}^{*} \mathcal{D}_{\omega} \Gamma+h_{1}-d_{2} \varepsilon^{4 m-4} \Delta_{0}^{2}|z|^{6-4 m}\right\|_{C_{-1-2 m}^{0, \alpha}}=d_{2} \varepsilon^{4 m-4}\left\|\left(\mathcal{D}_{\omega}^{*} \mathcal{D}_{\omega}-\Delta_{0}^{2}\right)|z|^{6-4 m}\right\|_{C_{-1-2 m}^{0, \alpha}}$.

On the annulus $A_{r}$ we have

$$
\begin{aligned}
\left\|\left(\mathcal{D}_{\omega}^{*} \mathcal{D}_{\omega}-\Delta_{0}^{2}\right)|z|^{6-4 m}\right\|_{C_{-1-2 m}^{0, \alpha}} & \leqslant\|\varphi\|_{C_{2}^{4, \alpha}}\left\||z|^{6-4 m}\right\|_{C_{3-2 m}^{4, \alpha}} \\
& \leqslant C r^{2} \cdot r^{3-2 m},
\end{aligned}
$$

where $\varphi=O\left(|z|^{4}\right)$. We have $r \geqslant r_{\varepsilon}$, and so

$$
\varepsilon^{4 m-4} r^{5-2 m}=O\left(\varepsilon^{\kappa}\right)
$$

for some $\kappa>2 m$, since $\alpha<1$.

We also have

$$
Q_{\omega}(\Gamma)=Q_{\omega}\left(d_{0} \varepsilon^{2 m-2}|z|^{4-2 m}\right)+\left[Q_{\omega}(\Gamma)-Q_{\omega}\left(d_{0} \varepsilon^{2 m-2}|z|^{4-2 m}\right)\right] .
$$

The next highest order term in $\Gamma$ after $d_{0} \varepsilon^{2 m-2}|z|^{4-2 m}$ is $d_{1} \varepsilon^{2 m}|z|^{2-2 m}$, so on the annulus $A_{r}$

$$
\begin{aligned}
\left\|Q_{\omega}(\Gamma)-Q_{\omega}\left(d_{0} \varepsilon^{2 m-2}|z|^{4-2 m}\right)\right\|_{C_{-1-2 m}^{0, \alpha}} & \leqslant C \varepsilon^{4 m}\left\||z|^{2-2 m}\right\|_{C_{2}^{4, \alpha}}\left\||z|^{2-2 m}\right\|_{C_{3-2 m}^{4, \alpha}} \\
& \leqslant C \varepsilon^{4 m} r^{-2 m} r^{-1}=O\left(\varepsilon^{\kappa}\right),
\end{aligned}
$$

for $\kappa>2 m$, since

$$
\alpha<\frac{2 m}{2 m+1}
$$


What remains is to estimate

$$
Q_{\omega}\left(d_{0} \varepsilon^{2 m-2}|z|^{4-2 m}\right)-\Delta_{0}^{2}\left(d_{2} \varepsilon^{4 m-4}|z|^{6-4 m}\right) .
$$

It is not hard to check that both terms are of the same order, and for $m>3$ they are sufficiently small. However for $m=3$ we need to work harder. On the annulus $A_{r}$, using Lemma 18, we have

$$
\begin{gathered}
\left\|Q_{\omega}\left(d_{0} \varepsilon^{2 m-2}|z|^{4-2 m}\right)-Q_{0}\left(d_{0} \varepsilon^{2 m-2}|z|^{4-2 m}\right)\right\|_{C_{-1-2 m}^{0, \alpha}} \leqslant \\
\leqslant C r^{2} \varepsilon^{4 m-4}\left\||z|^{4-2 m}\right\|_{C_{2}^{4, \alpha}}\left\||z|^{4-2 m}\right\|_{C_{3-2 m}^{4, \alpha}} \\
\leqslant C \varepsilon^{4 m-4} r^{5-2 m}=O\left(\varepsilon^{\kappa}\right),
\end{gathered}
$$

for $\kappa>2 m$, where $Q_{0}$ is given by the flat metric. Using that $\varepsilon^{2} \eta$ is scalar flat, we have

$0=\mathbf{s}\left[i \partial \bar{\partial}\left(\frac{|z|^{2}}{2}+d_{0} \varepsilon^{2 m-2}|z|^{4-2 m}+d_{1} \varepsilon^{2 m}|z|^{2-2 m}+d_{2} \varepsilon^{4 m-4}|z|^{6-4 m}+\varepsilon^{2} \psi_{4-4 m}\left(\varepsilon^{-1} z\right)\right)\right]$,

so if we write $\varepsilon^{2} \eta=i \partial \bar{\partial}\left(\frac{|z|^{2}}{2}+\varepsilon^{2} \psi\left(\varepsilon^{-1} z\right)\right)$, then we get

$$
\begin{aligned}
-\Delta_{0}^{2}\left(d_{2} \varepsilon^{4 m-4}|z|^{6-4 m}\right)+ & Q_{0}\left(d_{0} \varepsilon^{2 m-2}|z|^{4-2 m}\right)=\Delta_{0}^{2}\left(\varepsilon^{2} \psi_{4-4 m}\left(\varepsilon^{-1} z\right)\right)+ \\
& +Q_{0}\left(d_{0} \varepsilon^{2 m-2}|z|^{4-4 m}\right)-Q_{0}\left(\varepsilon^{2} \psi\left(\varepsilon^{-1} z\right)\right)
\end{aligned}
$$

and so on $A_{r}$ we have

$$
\begin{aligned}
\|-\Delta_{0}^{2}\left(d_{2} \varepsilon^{4 m-4}|z|^{6-4 m}\right)+ & Q_{0}\left(d_{0} \varepsilon^{2 m-2}|z|^{4-2 m}\right)\left\|_{C_{-1-2 m}^{0, \alpha}} \leqslant C \varepsilon^{4 m-2}\right\||z|^{4-4 m} \|_{C_{3-2 m}^{4, \alpha}}+ \\
& +C \varepsilon^{4 m}\left\||z|^{2-2 m}\right\|_{C_{2}^{4, \alpha}}\left\||z|^{2-2 m}\right\|_{C_{3-2 m}^{4, \alpha}} \\
\leqslant & C \varepsilon^{4 m-2} r^{1-2 m}+C \varepsilon^{4 m} r^{-1-2 m}=O\left(\varepsilon^{\kappa}\right),
\end{aligned}
$$

for $\kappa>2 m$, where we used that the largest order term in $\varepsilon^{2} \psi\left(\varepsilon^{-1} z\right)$ after the leading term is $\varepsilon^{2 m}|z|^{2-2 m}$. Combining all these estimates, we obtain the required bound (27).

Now to deal with modifying $\varepsilon^{2} \eta$, we have the following.

Lemma 25. We can find a function $\Psi$ on $\mathrm{Bl}_{0} \mathbf{C}^{m}$ of the form

$$
\Psi=A_{4}(z)+A_{5}(z)+\Psi_{1},
$$

where

and in addition

$$
\left\|\Psi_{1}\right\|_{C_{3-2 m}^{k, \alpha}\left(B_{2 r_{\varepsilon}} \backslash B_{r_{\varepsilon}}\right)}=O\left(\varepsilon^{\kappa}\right)
$$

$$
\left\|\mathbf{s}\left(\varepsilon^{2} \eta+i \partial \bar{\partial} \Psi\right)-\mathbf{s}(p)\right\|_{C_{-1-2 m}^{0, \alpha}\left(B_{r_{\varepsilon}}\right)}=O\left(\varepsilon^{\kappa}\right)
$$

for some $\kappa>2 m$.

Proof. We will work in terms of $\eta$ with the variable $w=\varepsilon^{-1} z$. Write $R_{\varepsilon}=\varepsilon^{-1} r_{\varepsilon}$, so that in terms of $w$, we are gluing on the annulus $B_{2 R_{\varepsilon}} \backslash B_{R_{\varepsilon}}$.

Write $\widetilde{A}_{4}(w), \widetilde{A}_{5}(w)$ for the functions $A_{4}(w)$ and $A_{5}(w)$ cut off on the annulus $B_{4 R_{\varepsilon}} \backslash B_{2 R_{\varepsilon}}$. Since

we have

$$
\left\|\varepsilon^{2} \widetilde{A}_{4}+\varepsilon^{3} \widetilde{A}_{5}\right\|_{C_{2}^{4, \alpha}} \leqslant C \varepsilon^{2} R_{\varepsilon}^{2} \ll 1
$$

$$
\left\|Q_{\eta}\left(\varepsilon^{2} \widetilde{A}_{4}+\varepsilon^{3} \widetilde{A}_{5}\right)\right\|_{C_{0}^{0, \alpha}} \leqslant C\left(\varepsilon^{2} R_{\varepsilon}^{2}\right)\left(\varepsilon^{2}\right)=C \varepsilon^{4} R_{\varepsilon}^{2}
$$


It follows from Proposition 14 that we can find $\widetilde{\Psi}$ such that

$$
\mathcal{D}_{\eta}^{*} \mathcal{D}_{\eta} \widetilde{\Psi}=Q_{\eta}\left(\varepsilon^{2} \widetilde{A}_{4}+\varepsilon^{3} \widetilde{A}_{5}\right),
$$

and

$$
\|\widetilde{\Psi}\|_{C_{4}^{4, \alpha}} \leqslant C \varepsilon^{4} R_{\varepsilon}^{2}=C \varepsilon^{2} r_{\varepsilon}^{2}
$$

Setting $\Psi_{1}(z)=\varepsilon^{2} \widetilde{\Psi}\left(\varepsilon^{-1} z\right)$, we then have

$$
\left\|\Psi_{1}\right\|_{C_{3-2 m}^{4, \alpha}\left(B_{2 r_{\varepsilon}} \backslash B_{r_{\varepsilon}}\right)} \leqslant C r_{\varepsilon}^{2 m+1}\left\|\Psi_{1}\right\|_{C_{4}^{4, \alpha}\left(B_{2 r_{\varepsilon}} \backslash B_{r_{\varepsilon}}\right)} \leqslant C r_{\varepsilon}^{2 m+1} r_{\varepsilon}^{2}=O\left(\varepsilon^{\kappa}\right),
$$

for $\kappa>2 m$, since

In addition, using Lemma 22,

$$
\alpha>\frac{2 m}{2 m+3}
$$

$$
\begin{aligned}
& \mathbf{s}\left(\eta+i \partial \bar{\partial}\left(\varepsilon^{2} \widetilde{A}_{4}+\varepsilon^{3} \widetilde{A}_{5}+\widetilde{\Psi}\right)\right)=L_{\eta}\left(\varepsilon^{2} \widetilde{A}_{4}+\varepsilon^{3} \widetilde{A}_{5}+\widetilde{\Psi}\right)+Q_{\eta}\left(\varepsilon^{2} \widetilde{A}_{4}+\varepsilon^{3} \widetilde{A}_{5}+\widetilde{\Psi}\right) \\
&= \varepsilon^{2} \mathbf{s}(p)+L_{\eta}(\widetilde{\Psi})+\left(L_{\eta}+\Delta_{0}^{2}\right)\left(\varepsilon^{2} \widetilde{A}_{4}+\varepsilon^{3} \widetilde{A}_{5}\right) \\
&+Q_{\eta}\left(\varepsilon^{2} \widetilde{A}_{4}+\varepsilon^{3} \widetilde{A}_{5}\right) \\
&+Q_{\eta}\left(\varepsilon^{2} \widetilde{A}_{4}+\varepsilon^{3} \widetilde{A}_{5}+\widetilde{\Psi}\right)-Q_{\eta}\left(\varepsilon^{2} \widetilde{A}_{4}+\varepsilon^{4} \widetilde{A}_{5}\right)
\end{aligned}
$$

Using the equality $\mathcal{D}_{\eta}^{*} \mathcal{D}_{\eta}=L_{\eta}$ we have $L_{\eta}(\widetilde{\Psi})+Q_{\eta}\left(\varepsilon^{2} \widetilde{A}_{4}+\varepsilon^{3} \widetilde{A}_{5}\right)=0$. Using the fact that $\eta$ differs from the flat metric by order $|w|^{2-2 m}$, we get

$\left\|\mathbf{s}\left(\eta+i \partial \bar{\partial}\left(\varepsilon^{2} \widetilde{A}_{4}+\varepsilon^{3} \widetilde{A}_{5}+\widetilde{\Psi}\right)\right)-\varepsilon^{2} \mathbf{s}(p)\right\|_{C_{-1-2 m}^{0, \alpha}\left(B_{2 R_{\varepsilon}}\right)} \leqslant C\left(\varepsilon^{2} R_{\varepsilon}^{3}+\varepsilon^{8} R_{\varepsilon}^{7+2 m}\right)=O\left(\varepsilon^{1+\delta}\right)$, for some $\delta>0$. Since on the ball $B_{R_{\varepsilon}}$ in terms of $w$ (and on $B_{r_{\varepsilon}}$ in terms of $z$ ) we have

$$
\mathbf{s}\left(\varepsilon^{2} \eta+i \partial \bar{\partial} \Psi\right)=\varepsilon^{-2} \mathbf{s}\left(\eta+i \partial \bar{\partial}\left(\varepsilon^{2} \widetilde{A}_{4}+\varepsilon^{3} \widetilde{A}_{5}+\widetilde{\Psi}\right)\right),
$$

it follows that

$$
\left\|\mathbf{s}\left(\varepsilon^{2} \eta+i \partial \bar{\partial} \Psi\right)-\mathbf{s}(p)\right\|_{C_{-1-2 m}^{0, \alpha}\left(B_{r_{\varepsilon}}\right)}=O\left(\varepsilon^{\kappa}\right)
$$

for some $\kappa>2 m$.

We now define our new approximate metric $\Omega_{1}$ to be equal to $\omega+i \partial \bar{\partial} \Gamma$ on $M \backslash B_{2 r_{\varepsilon}}$, equal to $\varepsilon^{2} \eta+i \partial \bar{\partial} \Psi$ on $B_{r_{\varepsilon}}$, and on the annular region $B_{2 r_{\varepsilon}} \backslash B_{r_{\varepsilon}}$ we let

$$
\begin{aligned}
\Omega_{1}= & i \partial \bar{\partial}\left(\frac{|z|^{2}}{2}+A_{4}+A_{5}+\gamma_{1} \varphi_{6}+\gamma_{1} \Gamma_{1}+d_{0} \varepsilon^{2 m-2}|z|^{4-2 m}+d_{1} \varepsilon^{2 m}|z|^{2-2 m}\right. \\
& \left.+d_{2} \varepsilon^{4 m-4}|z|^{6-4 m}+\gamma_{2} \varepsilon^{2} \psi_{4-4 m}\left(\varepsilon^{-1} z\right)+\gamma_{2} \Psi_{1}\right) .
\end{aligned}
$$

Lemma 26. On the annular region $B_{2 r_{\varepsilon}} \backslash B_{r_{\varepsilon}}$ we have

$$
\left\|\mathbf{s}\left(\Omega_{1}\right)-\mathbf{s}(p)\right\|_{C_{-1-2 m}^{0, \alpha}\left(B_{2 r_{\varepsilon}} \backslash B_{r_{\varepsilon}}\right)}=O\left(\varepsilon^{\kappa}\right),
$$

for some $\kappa>2 m$.

Proof. We compute the scalar curvature of $\Omega_{1}$ as a perturbation of the metric

$$
\omega_{0}=i \partial \bar{\partial}\left(\frac{|z|^{2}}{2}+d_{0} \varepsilon^{2 m-2}|z|^{4-2 m}+d_{1} \varepsilon^{2 m}|z|^{2-2 m}+d_{2} \varepsilon^{4 m-4}|z|^{6-4 m}\right)
$$

on the annulus $B_{2 r_{\varepsilon}} \backslash B_{r_{\varepsilon}}$. Since

$$
\mathbf{s}\left(\omega_{0}+\varepsilon^{2} i \partial \bar{\partial} \psi_{4-4 m}\left(\varepsilon^{-1} z\right)\right)=\mathbf{s}\left(\varepsilon^{2} \eta\right)=0,
$$


we have

$$
\begin{aligned}
\mathbf{s}\left(\omega_{0}\right) & =-\varepsilon^{2} L_{\omega_{0}}\left(\psi_{4-4 m}\left(\varepsilon^{-1} z\right)\right)-Q_{\omega_{0}}\left(\varepsilon^{2} \psi_{4-4 m}\left(\varepsilon^{-1} z\right)\right) \\
& =\varepsilon^{2} \Delta_{0}^{2}\left(\psi_{4-4 m}\left(\varepsilon^{-1} z\right)\right)+\text { lower order terms. }
\end{aligned}
$$

It follows that

$$
\left\|\mathbf{s}\left(\omega_{0}\right)\right\|_{C_{-1-2 m}^{0, \alpha}\left(B_{2 r_{\varepsilon}} \backslash B_{r_{\varepsilon}}\right)}=O\left(\varepsilon^{4 m-2} r_{\varepsilon}^{1-2 m}\right)=O\left(\varepsilon^{\kappa}\right)
$$

for some $\kappa>2 m$. Then

$$
\begin{aligned}
\mathbf{s}\left(\Omega_{1}\right)= & \mathbf{s}\left(\omega_{0}\right)+L_{\omega_{0}}\left(A_{4}+A_{5}+\gamma_{1} \varphi_{6}+\gamma_{1} \Gamma_{1}+\gamma_{2} \varepsilon^{2} \psi_{4-4 m}\left(\varepsilon^{-1} z\right)+\gamma_{2} \Psi_{1}\right) \\
& +Q_{\omega_{0}}\left(A_{4}+A_{5}+\gamma_{1} \varphi_{6}+\gamma_{1} \Gamma_{1}+\gamma_{2} \varepsilon^{2} \psi_{4-4 m}\left(\varepsilon^{-1} z\right)+\gamma_{2} \Psi_{1}\right) .
\end{aligned}
$$

On the annulus $B_{2 r_{\varepsilon}} \backslash B_{r_{\varepsilon}}$ we have

$$
A_{4}+A_{5}+\gamma_{1} \varphi_{6}+\gamma_{1} \Gamma_{1}+\gamma_{2} \varepsilon^{2} \psi_{4-4 m}\left(\varepsilon^{-1} z\right)+\gamma_{2} \Psi_{1}=A_{4}+\text { lower order terms, }
$$
and also

$$
\begin{aligned}
\left\|\gamma_{1} \varphi_{6}+\gamma_{1} \Gamma_{1}+\gamma_{2} \varepsilon^{2} \psi_{4-4 m}\left(\varepsilon^{-1} z\right)+\gamma_{2} \Psi_{1}\right\|_{C_{3-2 m}^{4, \alpha}} & =O\left(r_{\varepsilon}^{2 m+3}+\varepsilon^{4 m-2} r_{\varepsilon}^{1-2 m}+\varepsilon^{\kappa}\right) \\
& =O\left(\varepsilon^{\kappa}\right),
\end{aligned}
$$

for some $\kappa>2 m$, and

It follows that

$$
\Delta_{0}^{2}\left(A_{4}+A_{5}\right)=-\mathbf{s}(p) .
$$

$$
\begin{aligned}
\left\|\mathbf{s}\left(\Omega_{1}\right)-\mathbf{s}(p)\right\|_{C_{-1-2 m}^{0, \alpha}} \leqslant & \left\|\left(L_{\omega_{0}}+\Delta_{0}^{2}\right)\left(A_{4}+A_{5}\right)\right\|_{C_{-1-2 m}^{0, \alpha}}+O\left(\varepsilon^{\kappa}\right) \\
& +C\left\|A_{4}\right\|_{C_{2}^{4, \alpha}}\left\|A_{4}\right\|_{C_{3-2 m}^{4, \alpha}} \\
= & O\left(\varepsilon^{\kappa}\right)
\end{aligned}
$$

for some $\kappa>2 m$.

Let us write $\Omega_{1}=\omega_{\varepsilon}+i \partial \bar{\partial} u_{1}$. From the results above, we find

Lemma 27. We have

$$
\left\|\mathbf{s}\left(\Omega_{1}\right)-\mathbf{l}_{\Omega_{1}}\left(\mathbf{s}+h_{1}\right)\right\|_{C_{-1-2 m}^{0, \alpha}}=O\left(\varepsilon^{\kappa}\right),
$$

for some $\kappa>2 m$, and

$$
\left\|u_{1}\right\|_{C_{3}^{k, \alpha}}=O\left(\varepsilon^{\delta}\right)
$$

for some $\delta>0$.

Proof. We work on the regions $M \backslash B_{2 r_{\varepsilon}}$ and $B_{2 \varepsilon}$ separately. On $M \backslash B_{2 r_{\varepsilon}}$ we have $u_{1}=\Gamma, \omega_{\varepsilon}=\omega$ and $\mathbf{l}\left(h_{1}\right)=h_{1}$, so the result follows from Lemma 24, together with

$$
\left\|\mathbf{l}_{\Omega_{1}}\left(h_{1}\right)-h_{1}\right\|_{C_{-1-2 m}^{0, \alpha}}=\left\|\frac{1}{2} \nabla \Gamma \cdot \nabla h_{1}\right\|_{C_{-1-2 m}^{0, \alpha}} \leqslant C \varepsilon^{4 m-4}=O\left(\varepsilon^{\kappa}\right),
$$

for $\kappa>2 m$, since $m>2$.

On $B_{2 r_{\varepsilon}}$ we have

$$
\left\|\mathbf{l}_{\Omega_{1}}\left(h_{1}\right)\right\|_{C_{-1-2 m}^{0, \alpha}} \leqslant C r_{\varepsilon}^{2 m+1}\left\|\mathbf{l}_{\Omega_{1}}\left(h_{1}\right)\right\|_{C_{0}^{0, \alpha}} \leqslant C r_{\varepsilon}^{2 m+1} \varepsilon^{2 m-2}=O\left(\varepsilon^{\kappa}\right)
$$

for some $\kappa>2 m$, since $\alpha>\frac{2}{2 m+1}$. In addition

$$
\left\|\mathbf{l}_{\Omega_{1}}(\mathbf{s}-\mathbf{s}(p))\right\|_{C_{-1-2 m}^{0, \alpha}} \leqslant C r_{\varepsilon}^{2 m+3}\left\|\mathbf{l}_{\Omega_{1}}(\mathbf{s}-\mathbf{s}(p))\right\|_{C_{2}^{0, \alpha}} \leqslant C r_{\varepsilon}^{2 m+3}=O\left(\varepsilon^{\kappa}\right),
$$

for some $\kappa>2 m$. The bound (29) then follows from Lemmas 25 and 26 . 
As for (30), note that outside $B_{2 r_{\varepsilon}}$ the leading term in $u_{1}$ is of order $\varepsilon^{2 m-2}|z|^{4-2 m}$, while inside $B_{2 r_{\varepsilon}}$ the leading term is of order $|z|^{4}$. The bound (30) is then easy to check.

3.11. The approximate solution $\Omega_{2}$. We need to modify $\Omega_{1}$ once more to obtain a metric $\Omega_{2}=\Omega_{1}+i \partial \bar{\partial} u_{2}$. For this we want to find $\left(u_{2}, h_{2}\right)$ solving

$$
-\mathcal{D}_{\Omega_{1}}^{*} \mathcal{D}_{\Omega_{1}} u_{2}-\mathbf{l}_{\Omega_{1}}\left(h_{2}\right)=\mathbf{l}_{\Omega_{1}}\left(\mathbf{s}+h_{1}\right)-\mathbf{s}\left(\Omega_{1}\right) .
$$

We will use the inverse operator from Proposition 20 , so in addition to the estimate from Lemma 27, we need to bound the integral of $\mathbf{l}_{\Omega_{1}}\left(\mathbf{s}+h_{1}\right)-\mathbf{s}\left(\Omega_{1}\right)$. For this we have the following.

Lemma 28. We have

$$
\int_{\mathrm{Bl}_{p} M} \mathbf{l}_{\Omega_{1}}\left(\mathbf{s}+h_{1}\right)-\mathbf{s}\left(\Omega_{1}\right) \frac{\Omega_{1}^{m}}{m !}=O\left(\varepsilon^{\kappa}\right),
$$

for some $\kappa>2 m$.

Proof. First note that from Lemma 10] we have

$$
\int_{\mathrm{Bl}_{p} M} \mathbf{l}_{\Omega_{1}}(\mathbf{s})-\mathbf{s}\left(\Omega_{1}\right) \frac{\Omega_{1}^{m}}{m !}=\int_{\mathrm{Bl}_{p} M} \mathbf{l}(\mathbf{s})-\mathbf{s}\left(\omega_{\varepsilon}\right) \frac{\omega_{\varepsilon}^{m}}{m !},
$$

since $\mathbf{s} \in \overline{\mathfrak{t}}$ and the total scalar curvature is an invariant of the Kähler class.

We have

$$
\begin{aligned}
\int_{\mathrm{Bl}_{p} M} \mathbf{l}_{\Omega_{1}}\left(h_{1}\right) \Omega_{1}^{m}-\int_{\mathrm{Bl}_{p} M} \mathbf{l}\left(h_{1}\right) \omega_{\varepsilon}^{m}= & \int_{\mathrm{Bl}_{p} M} \frac{1}{2} \nabla \mathbf{l}\left(h_{1}\right) \cdot \nabla u_{1} \Omega_{1}^{m}+ \\
& +\int_{\mathrm{Bl}_{p} M} \mathbf{l}\left(h_{1}\right)\left(\Omega_{1}^{m}-\omega_{\varepsilon}^{m}\right) .
\end{aligned}
$$

On $M \backslash B_{1}$, we can bound this by $\left|h_{1}\right|\left\|u_{1}\right\|_{C_{2}^{4, \alpha}}$, as in Equation (21). We have

$$
\left|h_{1}\right|\left\|u_{1}\right\|_{C^{4, \alpha}\left(M \backslash B_{1}\right)} \leqslant C \varepsilon^{2 m-2} \varepsilon^{2 m-2}=O\left(\varepsilon^{\kappa}\right),
$$

for some $\kappa>2 m$ if $m>2$. To bound (32) on $B_{1} \backslash B_{2 r_{\varepsilon}}$, note that

$$
\left\|u_{1}\right\|_{C_{4-2 m}^{4, \alpha}\left(M \backslash B_{2 r_{\varepsilon}}\right)}=O\left(\varepsilon^{2 m-2}\right),
$$

and $\left|\mathbf{l}\left(h_{1}\right)\right|+\left|\nabla \mathbf{l}\left(h_{1}\right)\right| \leqslant C \varepsilon^{2 m-2}$ from Lemma 15. It follows that

$$
\left\|\mathbf{l}\left(h_{1}\right)\left(\Omega_{1}^{m}-\omega_{\varepsilon}^{m}\right)\right\|_{C_{4-2 m}^{0}\left(B_{1} \backslash B_{2 r_{\varepsilon}}\right)} \leqslant C \varepsilon^{4 m-4} .
$$

The integral on $B_{1} \backslash B_{2 r_{\varepsilon}}$ is then bounded by

Similarly

$$
\int_{2 r_{\varepsilon}}^{1} \varepsilon^{4 m-4} r^{2-2 m} r^{2 m-1} d r \leqslant C \varepsilon^{4 m-4} .
$$

$$
\left\|\nabla \mathbf{l}\left(h_{1}\right) \cdot \nabla u_{1}\right\|_{C_{3-2 m}^{0}} \leqslant C \varepsilon^{4 m-4}
$$

so the other term in (32) is also bounded by $\varepsilon^{4 m-4}$. Since $m>2$, we have $\varepsilon^{4 m-4}=$ $O\left(\varepsilon^{\kappa}\right)$ for some $\kappa>2 m$.

The lift $\mathbf{l}\left(h_{1}\right)$ equals $h_{1}$ outside $B_{2 r_{\varepsilon}}$, and the volume of $B_{2 r_{\varepsilon}}$ is of order $r_{\varepsilon}^{2 m}$, so we have

$$
\int_{\mathrm{Bl}_{p} M} \mathbf{l}\left(h_{1}\right) \frac{\omega_{\varepsilon}^{m}}{m !}=\int_{M} h_{1} \frac{\omega^{m}}{m !}+O\left(\varepsilon^{2 m-2} r_{\varepsilon}^{2 m}\right)=\int_{M} h_{1} \frac{\omega^{m}}{m !}+O\left(\varepsilon^{\kappa}\right),
$$


for some $\kappa>2 m$.

Since $\mathbf{s} \in \mathfrak{t}$, we can relate the integrals on $\mathrm{Bl}_{p} M$ and on $M$ using the formulas in Proposition 35. We have

$$
\int_{\mathrm{Bl}_{p} M} \mathbf{l}(\mathbf{s}) \frac{\omega_{\varepsilon}^{m}}{m !}=\int_{M} \mathbf{s} \frac{\omega^{m}}{m !}-\frac{\varepsilon^{2 m}}{m !} \mathbf{s}(p)+O\left(\varepsilon^{\kappa}\right),
$$

for some $\kappa>2 m$, and also

$$
\int_{\mathrm{Bl}_{p} M} \mathbf{s}\left(\omega_{\varepsilon}\right) \frac{\omega_{\varepsilon}^{m}}{m !}=\int_{M} \mathbf{s} \frac{\omega^{m}}{m !}-\frac{2 \pi \varepsilon^{2 m-2}}{(m-2) !} .
$$

Combining these formulas, we have

$$
\int_{\mathrm{Bl}_{p} M} \mathbf{l}\left(\mathbf{s}+h_{1}\right)-\mathbf{s}\left(\omega_{\varepsilon}\right) \frac{\omega_{\varepsilon}^{m}}{m !}=\int_{M} h_{1} \frac{\omega^{m}}{m !}+\frac{2 \pi \varepsilon^{2 m-2}}{(m-2) !}-\frac{\varepsilon^{2 m}}{m !} \mathbf{s}(p)+O\left(\varepsilon^{\kappa}\right) .
$$

From the formula for $h_{1}$ in Lemma 24, the first 3 terms on the right cancel, leaving only $O\left(\varepsilon^{\kappa}\right)$.

Letting $C$ be the average of $\mathbf{l}_{\Omega_{1}}\left(\mathbf{s}+h_{1}\right)-\mathbf{s}\left(\Omega_{1}\right)$ with respect to $\Omega_{1}$, we can apply Proposition 20 to find $u_{2}$ and $h_{2}^{\prime} \in \overline{\mathfrak{h}}_{0}$ such that

$$
-\mathcal{D}_{\Omega_{1}}^{*} \mathcal{D}_{\Omega_{1}} u_{2}-\mathbf{l}_{\Omega_{1}}\left(h_{2}^{\prime}\right)=\mathbf{l}_{\Omega_{1}}\left(\mathbf{s}+h_{1}\right)-\mathbf{s}\left(\Omega_{1}\right)-C .
$$

Letting $h_{2}=h_{2}^{\prime}-C$, this implies that we can solve (31), with

$$
\left\|u_{2}\right\|_{C_{3-2 m}^{4, \alpha}}+\left|h_{2}\right|=O\left(\varepsilon^{\kappa}\right)
$$

with $\kappa>2 m$. We now let $\Omega_{2}=\Omega_{1}+i \partial \bar{\partial} u_{2}$. This satisfies the following.

Lemma 29.

$$
\left\|\mathbf{s}\left(\Omega_{2}\right)-\mathbf{l}_{\Omega_{2}}\left(\mathbf{s}+h_{1}+h_{2}\right)\right\|_{C_{-2 m}^{0, \alpha}}=O\left(\varepsilon^{\kappa}\right)
$$

for some $\kappa>2 m$.

Proof. First we have

$$
\begin{aligned}
\mathbf{s}\left(\Omega_{2}\right)= & \mathbf{s}\left(\Omega_{1}\right)+L_{\Omega_{1}}\left(u_{2}\right)+Q_{\Omega_{1}}\left(u_{2}\right) \\
= & \mathbf{s}\left(\Omega_{1}\right)-\mathcal{D}_{\Omega_{1}}^{*} \mathcal{D}_{\Omega_{1}}\left(u_{2}\right)+\frac{1}{2} \nabla \mathbf{s}\left(\Omega_{1}\right) \cdot \Omega_{1} \nabla u_{2}+Q_{\Omega_{1}}\left(u_{2}\right) \\
= & \mathbf{l}_{\Omega_{1}}\left(\mathbf{s}+h_{1}+h_{2}\right)+\frac{1}{2} \nabla \mathbf{s}\left(\Omega_{1}\right) \cdot \Omega_{1} \nabla u_{2}+Q_{\Omega_{1}}\left(u_{2}\right) \\
= & \mathbf{l}_{\Omega_{2}}\left(\mathbf{s}+h_{1}+h_{2}\right)-\frac{1}{2} \nabla \mathbf{l}\left(\mathbf{s}+h_{1}+h_{2}\right) \cdot \nabla u_{2} \\
& \quad+\frac{1}{2} \nabla \mathbf{s}\left(\Omega_{1}\right) \cdot \Omega_{1} \nabla u_{2}+Q_{\Omega_{1}}\left(u_{2}\right),
\end{aligned}
$$

where by $\Omega_{1}$ we indicate that the gradients and inner products are taken with respect to $\Omega_{1}$ instead of $\omega_{\varepsilon}$. We need to estimate $\nabla \mathbf{s}\left(\Omega_{1}\right)$, and for this we have

$$
\left\|\mathbf{s}\left(\Omega_{1}\right)-\mathbf{s}\left(\omega_{\varepsilon}\right)\right\|_{C_{-1}^{1, \alpha}}=\left\|L_{\omega^{\prime}}\left(u_{1}\right)\right\|_{C_{-1}^{1, \alpha}} \leqslant C\left\|u_{1}\right\|_{C_{3}^{5, \alpha}} \leqslant C
$$

where $\omega^{\prime}=\omega_{\varepsilon}+t i \partial \bar{\partial} u_{1}$ for some $t \in[0,1]$, where we used (30). We then have

$$
\left\|\nabla \mathbf{l}\left(\mathbf{s}+h_{1}+h_{2}\right) \cdot \nabla u_{2}-\nabla \mathbf{s}\left(\Omega_{1}\right) \cdot \Omega_{1} \nabla u_{2}\right\|_{C_{-2 m}^{0, \alpha}} \leqslant C\left\|u_{2}\right\|_{C_{2-2 m}^{4, \alpha}}=O\left(\varepsilon^{\kappa}\right),
$$


by (33). So we only need to estimate $Q_{\Omega_{1}}\left(u_{2}\right)$, but for this Lemma 17 implies

$$
\begin{aligned}
\left\|Q_{\Omega_{1}}\left(u_{2}\right)\right\|_{C_{-2 m}^{0, \alpha}} & \leqslant C\left\|u_{2}\right\|_{C_{2}^{4, \alpha}}\left\|u_{2}\right\|_{C_{4-2 m}^{4, \alpha}} \\
& \leqslant C \varepsilon^{1-2 m}\left\|u_{2}\right\|_{C_{3-2 m}^{4, \alpha}} \varepsilon^{-1}\left\|u_{2}\right\|_{C_{3-2 m}^{4, \alpha}}=O\left(\varepsilon^{\kappa}\right),
\end{aligned}
$$

using (33) again.

3.12. Solving the non-linear equation. We are finally ready to try solving the equation we need to, i.e. we want $u, h$ such that

$$
\mathbf{s}\left(\Omega_{2}+i \partial \bar{\partial} u\right)=\mathbf{l}_{\Omega_{2}+i \partial \bar{\partial} u}\left(\mathbf{s}+h_{1}+h_{2}+h\right) .
$$

Expanding this in terms of the linearized operator, we have

$$
\begin{aligned}
\mathbf{s}\left(\Omega_{2}\right)+L_{\Omega_{2}}(u)+Q_{\Omega_{2}}(u)= & \mathbf{l}_{\Omega_{2}}\left(\mathbf{s}+h_{1}+h_{2}\right)+\frac{1}{2} \nabla u \cdot \nabla \mathbf{l}\left(\mathbf{s}+h_{1}+h_{2}\right) \\
& +\mathbf{l}_{\Omega_{2}}(h)+\frac{1}{2} \nabla u \cdot \nabla h,
\end{aligned}
$$

which we can write as

$$
\widetilde{G}_{1}(u, h)=\mathbf{l}_{\Omega_{2}}\left(\mathbf{s}+h_{1}+h_{2}\right)-\mathbf{s}\left(\Omega_{2}\right)+\frac{1}{2} \nabla u \cdot \nabla h-Q_{\Omega_{2}}(u),
$$

where $\widetilde{G}_{1}$ is defined by

$$
\widetilde{G}_{1}(u, h):=L_{\Omega_{2}}(u)-\frac{1}{2} \nabla u \cdot \nabla \mathbf{l}\left(\mathbf{s}+h_{1}+h_{2}\right)-\mathbf{l}_{\Omega_{2}}(h) .
$$

It follows from Remark 21] and Lemma 29 that this operator $\widetilde{G}_{1}$ is sufficiently close to the operator $G_{1}$ in Proposition 19 when $\varepsilon \ll 1$, so that the inverse $P_{1}$ from Proposition[19] can be used to obtain an inverse $\widetilde{P}_{1}$ for $\widetilde{G}_{1}$ with uniformly bounded norm. We are therefore trying to solve the fixed point problem

$$
(u, h)=\mathcal{N}(u, h),
$$

where

$$
\begin{aligned}
\mathcal{N}(u, h): C_{\delta}^{4, \alpha} \times \overline{\mathfrak{h}} & \rightarrow C_{\delta}^{4, \alpha} \times \overline{\mathfrak{h}} \\
(u, h) & \mapsto \widetilde{P}_{1}\left(\mathbf{l}_{\Omega_{2}}\left(\mathbf{s}+h_{1}+h_{2}\right)-\mathbf{s}\left(\Omega_{2}\right)+\frac{1}{2} \nabla u \cdot \nabla h-Q_{\Omega_{2}}(u)\right),
\end{aligned}
$$

and $\delta=4-2 m+\tau$ for sufficiently small $\tau>0$.

The following lemma is essentially identical to Lemma 23 in [32].

Lemma 30. There is a constant $c_{1}>0$ such that if

$$
\left\|v_{i}\right\|_{C_{2}^{4, \alpha}},\left|g_{i}\right|<c_{1}
$$

for $i=1,2$, then

$$
\left\|\mathcal{N}\left(v_{1}, g_{1}\right)-\mathcal{N}\left(v_{2}, g_{2}\right)\right\|_{C_{\delta}^{4, \alpha} \times \bar{h}} \leqslant \frac{1}{2}\left\|\left(v_{1}-v_{2}, g_{1}-g_{2}\right)\right\|_{C_{\delta}^{4, \alpha} \times \overline{\mathfrak{h}}} .
$$

We can now complete the proof of Theorem 12 Proof of Theorem 12, From Lemma 29] we have

$$
\|\mathcal{N}(0,0)\|_{C_{\delta}^{4, \alpha} \times \overline{\mathfrak{h}}} \leqslant c_{2} \varepsilon^{\kappa^{\prime}}
$$

for some $\kappa^{\prime}>2 m$. Define

$$
S=\left\{(v, g):\|v\|_{C_{\delta}^{4, \alpha}},|g| \leqslant 2 c_{2} \varepsilon^{\kappa^{\prime}}\right\} .
$$


If $(v, g) \in S$, then for sufficiently small $\varepsilon$ we have $|g|<c_{1}$ with the $c_{1}$ from Lemma 30 and also

$$
\|v\|_{C_{2}^{4, \alpha}} \leqslant C \varepsilon^{\delta-2}\|v\|_{C_{\delta}^{4, \alpha}} \leqslant 2 C c_{2} \varepsilon^{\kappa^{\prime}+\delta-2}<c_{1},
$$

for sufficiently small $\varepsilon$, since $\kappa^{\prime}+\delta-2>0$. It follows that

$$
\|\mathcal{N}(v, g)\| \leqslant\|\mathcal{N}(v, g)-\mathcal{N}(0,0)\|+\|\mathcal{N}(0,0)\| \leqslant \frac{1}{2}\|(v, g)\|+c_{2} \varepsilon^{\kappa^{\prime}} \leqslant 2 c_{2} \varepsilon^{\kappa^{\prime}},
$$

so $\mathcal{N}$ is a contraction mapping $S$ into itself. We can therefore find a fixed point $(u, h)$ of $\mathcal{N}$ in $S$, and this gives a solution of the equation

$$
\mathbf{s}\left(\Omega_{2}+i \partial \bar{\partial} u\right)=\mathbf{l}_{\Omega_{2}+i \partial \bar{\partial} u}\left(\mathbf{s}+h_{1}+h_{2}+h\right) .
$$

From Lemma 24, Equation (33) and the fact that $|h| \leqslant 2 c_{2} \varepsilon^{\kappa^{\prime}}$, we have

$$
\begin{aligned}
\mathbf{s}+h_{1}+h_{2}+h= & \mathbf{s}+h_{1}+O\left(\varepsilon^{\kappa}\right) \\
= & \mathbf{s}-\varepsilon^{2 m-2} \frac{2 \pi}{(m-2) !}\left(V^{-1}+\mu(p)\right)+\varepsilon^{2 m} \frac{\mathbf{s}(p)}{m !}\left(V^{-1}+\mu(p)\right) \\
& \quad-d_{1} \varepsilon^{2 m} \frac{2 \pi^{m}}{(m-2) !} \Delta \mu(p)+O\left(\varepsilon^{\kappa}\right),
\end{aligned}
$$

for some $\kappa>2 m$. This is what we wanted to prove. Since the solution is obtained using the contraction mapping principle, the solution will depend smoothly on the parameters. In particular when we perform this construction at the set of all $T$ invariant points in $M$, then the constant in $O\left(\varepsilon^{\kappa}\right)$ can be chosen to be uniform.

\section{Relative Stability of Blowups}

In this section we will give the proof of Theorem 2. Since we want to deal with Kähler manifolds which are not necessarily algebraic, we will reformulate a simple version of the usual theory of K-stability in the Kähler setting, which is more similar to Tian's original definition in 34 than to the more recent algebro-geometric approach of Donaldson [11].

4.1. Relative K-stability for Kähler manifolds. We will define relative Kstability for Kähler manifolds similarly to Tian's definition 34. Our definition will actually be simpler since we only consider test-configurations with smooth central fibers.

Definition 31. A (smooth) test-configuration for a Kähler manifold $(M, \omega)$ consists of a holomorphic submersion $\tau: \mathcal{X} \rightarrow \mathbf{C}$ together with a holomorphic lift $v$ of the vector field $\frac{\partial}{\partial \theta}$, satisfying the following properties:

(1) $\mathcal{X}$ admits a Kähler metric $\Omega$, for which $v$ is a Hamiltonian Killing field.

(2) The pair $\left(\tau^{-1}(1),\left.\Omega\right|_{\tau^{-1}(1)}\right)$ is isometric to $(M, \omega)$.

The vector field $v$ gives a Hamiltonian Killing field on the central fiber $\left(M_{0}, \omega_{0}\right)$, which we will denote by $v$ also. Let us write $h_{v}$ for its Hamiltonian function. The Futaki invariant of this vector field is defined (see Futaki [12, Calabi [7]) to be

$$
\operatorname{Fut}\left(M_{0},\left[\omega_{0}\right], v\right)=\int_{M_{0}} h_{v}\left(\overline{\mathbf{s}}-\mathbf{s}\left(\omega_{0}\right)\right) \omega_{0}^{n},
$$

where $\overline{\mathbf{s}}$ is the average of the scalar curvature of $\omega_{0}$. The notation indicates that the Futaki invariant does not depend on the particular metric chosen, only its Kähler class. 
For the definition of relative stability, we need to recall the extremal vector field defined by Futaki-Mabuchi 13. Let us write $\operatorname{Aut}_{0}(M)$ for the connected component of the identity in the automorphism group of $M$, and let $\widetilde{\operatorname{Aut}}_{0}(M)$ be the kernel of the map from $\operatorname{Aut}_{0}(M)$ to the Albanese torus of $M$. Finally, let $G \subset \widetilde{\operatorname{Aut}}_{0}(M)$ be a maximal compact subgroup. Suppose that $\omega$ is $G$-invariant. Then the action of $G$ is Hamiltonian with respect to $\omega$ (see e.g. LeBrun-Simanca [17]). This means that we can identify elements in the Lie algebra $\mathfrak{g}$ with their Hamiltonian functions, normalized to have zero mean. Let us write

$$
\mathbf{s}_{e x t}=\operatorname{proj}_{\mathfrak{g}} \mathbf{s}(\omega) \text {, }
$$

for the $L^{2}$-projection of the scalar curvature of $\omega$ onto $\mathfrak{g}$. The extremal vector field is the vector field corresponding to $\mathbf{s}_{e x t}$. The main result in 13 is that this vector field is independent of the choice of $G$-invariant metric $\omega$.

Suppose now that $T$ is a maximal torus in $G$. We say that the test-configuration $(\mathcal{X}, v)$ is compatible with $T$, if there is a Hamiltonian holomorphic $T$-action on $\mathcal{X}$ preserving the fibers, leaving $v$ invariant, and such that when restricted to $\tau^{-1}(1)$ it recovers the $T$-action on $M$. We define the modified Futaki invariant of such a testconfiguration as follows. As before, the central fiber of the test-configuration has an induced Hamiltonian Killing field $v$. Again, let $h_{v}$ be a Hamiltonian function for $v$, normalized to have zero mean. In addition, $\left(M_{0}, \omega_{0}\right)$ is equipped with a Hamiltonian $T$-action, and in particular the extremal vector field of $M$ induces a Hamiltonian holomorphic vector field $v_{e x t}$ on $M_{0}$, with a Hamiltonian function $h_{v_{\text {ext }}}$.

Definition 32. The modified Futaki invariant of the test-configuration is defined to be

$$
\operatorname{Fut}_{v_{e x t}}\left(M_{0},\left[\omega_{0}\right], v\right)=\int_{M_{0}} h_{v}\left(h_{v_{e x t}}-\mathbf{s}\left(\omega_{0}\right)\right) \omega_{0}^{n} .
$$

Note, in particular, that this coincides with the usual Futaki invariant, if $h_{v}$ and $h_{v_{\text {ext }}}$ are orthogonal.

Definition 33. We say that $(M, \omega)$ is K-semistable (with respect to smooth testconfigurations), if

$$
\operatorname{Fut}\left(M_{0},\left[\omega_{0}\right], v\right) \geqslant 0
$$

for all smooth test-configurations, compatible with a maximal torus $T$ as above. If in addition equality only holds if the central fiber $M_{0}$ is biholomorphic to $M$, then $(M, \omega)$ is K-stable. Relative K-stability is defined analogously with the modified Futaki invariant replacing the Futaki invariant.

The following proposition follows from the theorem of Chen-Tian 8 , that the modified Mabuchi energy is bounded below, if $M$ admits an extremal metric in the Kähler class $[\omega]$. For test-configurations with smooth central fibers it is fairly straight-forward to relate the modified Futaki invariant to the behavior of the modified Mabuchi functional. This is explained carefully in Tosatti [36] and ClarkeTipler [9]. As a consequence we have the following.

Proposition 34. If $M$ admits an extremal metric $\omega$, then $(M, \omega)$ is relatively K-semistable (with respect to smooth test-configurations).

Using the method of [27] and 29] we can improve the "semistability" to "stability", but first we need to study Futaki invariants on blowups. 
4.2. Futaki invariants on blowups. Suppose that $(M, \omega)$ is a Kähler manifold, and $v$ is a Hamiltonian holomorphic vector field on $M$, with Hamiltonian function $h_{v}$. If $p \in M$ is such that $v$ vanishes at $p$, then $v$ lifts to a holomorphic vector field $\hat{v}$ on the blowup $\mathrm{Bl}_{p} M$. Moreover, the lift is Hamiltonian with suitable choices of Kähler metric on the blowup, in the class $\pi^{*}[\omega]-\varepsilon^{2}[E]$. We need to compute the Futaki invariant

$$
\operatorname{Fut}\left(\mathrm{Bl}_{p} M, \pi^{*}[\omega]-\varepsilon^{2}[E], \hat{v}\right)
$$

in terms of the Futaki invariant on $M$. In the algebraic case this computation was done by Stoppa [28, and was refined in [32] (see also Della Vedova [10]). On Kähler surfaces the first term of the expansion was calculated by Li-Shi [19] under the assumption that we blow up a non-degenerate zero of the vector field. We can obtain the general result for Kähler manifolds using the simple observation that the difference

$$
\operatorname{Fut}\left(\mathrm{Bl}_{p} M, \pi^{*}[\omega]-\varepsilon^{2}[E], \hat{v}\right)-\operatorname{Fut}(M,[\omega], v)
$$

can essentially be computed in a neighborhood of $p$, since we can choose the metric on the blowup to coincide with the metric $\omega$ outside a neighborhood of $p$. Indeed this is what the metric $\omega_{\varepsilon}$ in Section 3.4 is like. We can also choose the Hamiltonian function $h_{\hat{v}}$ to coincide with $h_{v}$ outside a small ball $B$ around $p$, by choosing $h_{\hat{v}}=\mathbf{l}\left(h_{v}\right)$. We then have the following formulas.

Proposition 35. For sufficiently small $\varepsilon>0$ we have

$$
\begin{aligned}
\int_{M} \omega^{m}-\int_{\mathrm{Bl}_{p} M} \omega_{\varepsilon}^{m} & =\varepsilon^{2 m}, \\
\int_{M} h_{v} \omega^{m}-\int_{\mathrm{Bl}_{p} M} \mathbf{l}\left(h_{v}\right) \omega_{\varepsilon}^{m} & =\varepsilon^{2 m} h_{v}(p)+\frac{\varepsilon^{2 m+2}}{m+1} \Delta h_{v}(p), \\
\int_{M} \mathbf{s}(\omega) \omega^{m}-\int_{\mathrm{Bl}_{p} M} \mathbf{s}\left(\omega_{\varepsilon}\right) \omega_{\varepsilon}^{m} & =2 \pi m(m-1) \varepsilon^{2 m-2}, \\
\int_{M} h_{v} \mathbf{s}(\omega) \omega^{m}-\int_{\mathrm{Bl}_{p} M} \mathbf{l}\left(h_{v}\right) \mathbf{s}\left(\omega_{\varepsilon}\right) \omega_{\varepsilon}^{m} & =2 \pi m(m-1) \varepsilon^{2 m-2} h_{v}(p) \\
& +2 \pi(m-2) \varepsilon^{2 m} \Delta h_{v}(p) .
\end{aligned}
$$

Proof. Each of these formulas can be reduced to a calculation on the projective space $\mathbf{P}^{m}$. The first and third formulas can also be checked easily using the cohomological interpretations of the integrals. The formulas involving $h_{v}$ could also be approached using equivariant cohomology, but we will not pursue this.

In order to reduce the problem to a calculation on $\mathbf{P}^{m}$, note that each pair of integrals coincides outside a ball $B$ (in the notation of Section 3.4 we are taking $\left.B=B_{2 r_{\varepsilon}}\right)$. So for instance we have

$$
\int_{M} \omega^{m}-\int_{\mathrm{Bl}_{p} M} \omega_{\varepsilon}^{m}=\int_{B} \omega^{m}-\int_{\mathrm{Bl}_{p} B} \omega_{\varepsilon}^{m},
$$

with similar formulas for the other 3 integrals. Since $v$ vanishes at $p$, we can choose coordinates around $p$ in which $v$ is given by a linear transformation. For small $\varepsilon>0$, we can therefore choose a metric $\Omega$ on $\mathbf{P}^{m}$ in the class $c_{1}(\mathcal{O}(1))$, together with a holomorphic Killing field $V$ vanishing at a point $P \in \mathbf{P}^{m}$ (with Hamiltonian $\left.h_{V}\right)$, such that the data $\left(B_{2 r_{\varepsilon}}(P), \Omega, V, h_{V}\right)$ is equivalent in the obvious sense to 
the corresponding data $\left(B_{2 r_{\varepsilon}}(p), \omega, v, h_{v}\right)$. It follows from (36) together with the analogous formula on $\mathbf{P}^{m}$, that

$$
\int_{M} \omega^{m}-\int_{\mathrm{Bl}_{p} M} \omega_{\varepsilon}^{m}=\int_{\mathbf{P}^{m}} \Omega^{m}-\int_{\mathrm{Bl}_{P} \mathbf{P}^{m}} \Omega_{\varepsilon}^{m},
$$

where $\Omega_{\varepsilon}$ is a metric on $\mathrm{Bl}_{P} \mathbf{P}^{m}$ in the class $\pi^{*}[\Omega]-\varepsilon^{2}[E]$ constructed just like we constructed $\omega_{\varepsilon}$. The analogous formula holds for all of the differences that we need to compute in Equation (35). We have therefore reduced the problem to a calculation on $\mathbf{P}^{m}$.

On $\mathbf{P}^{m}$ one way to do the calculation would be to perform a computation in terms of toric geometry, since we can assume that $P$ is fixed by a maximal torus of automorphisms of $\mathbf{P}^{m}$. Alternatively, we can use an algebro-geometric calculation, since by continuity it is enough to deal with the case when $\varepsilon$ is rational. It is essentially this that we have already calculated in [32, Lemma 28]. The results of that Lemma, together with the calculations in [11, Proposition 2.2.2] imply the result we want.

Using this proposition we can compute the Futaki invariant on a blowup, extending [32, Corollary 29] to the Kähler case.

Corollary 36. Suppose that $h_{v}$ is normalized to have zero mean. For sufficiently small $\varepsilon>0$ we have an expression

$$
\operatorname{Fut}\left(\mathrm{Bl}_{p} M,\left[\omega_{\varepsilon}\right], \hat{v}\right)=\operatorname{Fut}(M,[\omega], v)+A_{\varepsilon} h_{v}(p)+B_{\varepsilon} \Delta h_{v}(p),
$$

where $A_{\varepsilon}=O\left(\varepsilon^{2 m-2}\right)$ and $B_{\varepsilon}=O\left(\varepsilon^{2 m}\right)$ are functions of $\varepsilon$ depending on $(M,[\omega])$. One can easily expand $A_{\varepsilon}, B_{\varepsilon}$ in terms of $\varepsilon$. In general

$$
\operatorname{Fut}\left(\mathrm{Bl}_{p} M,\left[\omega_{\varepsilon}\right], \hat{v}\right)=\operatorname{Fut}(M,[\omega], v)+2 \pi m(m-1) \varepsilon^{2 m-2} h_{v}(p)+O\left(\varepsilon^{2 m}\right) .
$$

Suppose that $\operatorname{Fut}(M,[\omega], v)=0$, and $m>2$. Then

$$
\begin{aligned}
\operatorname{Fut}\left(\mathrm{Bl}_{p} M,\left[\omega_{\varepsilon}\right], \hat{v}\right)=2 & \pi m(m-1) \varepsilon^{2 m-2} h_{v}(p) \\
& +\varepsilon^{2 m}\left(2 \pi(m-2) \Delta h_{v}(p)-\overline{\mathbf{s}} h_{v}(p)\right)+O\left(\varepsilon^{2 m+2}\right),
\end{aligned}
$$

where $\overline{\mathbf{s}}$ is the average scalar curvature of $(M, \omega)$. In addition if $h_{v}(p)=\Delta h_{v}(p)=$ 0 , then $\operatorname{Fut}\left(\mathrm{Bl}_{p} M,\left[\omega_{\varepsilon}\right], \hat{v}\right)=0$.

Proof. Let us write $\overline{\mathbf{s}}_{\varepsilon}$ for the average scalar curvature of $\omega_{\varepsilon}$. Then from (35) we have

$$
\begin{aligned}
\overline{\mathbf{s}}_{\varepsilon} & =\frac{\int_{\mathrm{Bl}_{p} M} \mathbf{s}\left(\omega_{\varepsilon}\right) \omega_{\varepsilon}^{m}}{\int_{\mathrm{Bl}_{p} M} \omega_{\varepsilon}^{m}}=\frac{\int_{M} \mathbf{s}(\omega) \omega^{m}-2 \pi m(m-1) \varepsilon^{2 m-2}}{\int_{M} \omega^{m}-\varepsilon^{2 m}} \\
& =\overline{\mathbf{s}}+O\left(\varepsilon^{2 m-2}\right),
\end{aligned}
$$

and

$$
\begin{aligned}
\int_{\mathrm{Bl}_{p} M} \mathbf{l}\left(h_{v}\right) \omega_{\varepsilon}^{m}= & -\varepsilon^{2 m} h_{v}(p)-\frac{\varepsilon^{2 m+2}}{m+1} \Delta h_{v}(p) \\
\int_{\mathrm{Bl}_{p} M} \mathbf{l}\left(h_{v}\right) \mathbf{s}\left(\omega_{\varepsilon}\right) \omega_{\varepsilon}^{m}= & \int_{M} h_{v} \mathbf{s}(\omega) \omega^{m}-2 \pi m(m-1) \varepsilon^{2 m-2} h_{v}(p) \\
& -2 \pi(m-2) \varepsilon^{2 m} \Delta h_{v}(p)
\end{aligned}
$$


Combining these, and using that

$$
\operatorname{Fut}(M,[\omega], v)=-\int h_{v} \mathbf{s}(\omega) \omega^{m}
$$

since $h_{v}$ has integral zero, we get

$$
\begin{aligned}
\operatorname{Fut}\left(\mathrm{Bl}_{p} M,\left[\omega_{\varepsilon}\right], \hat{v}\right)= & \int_{\mathrm{Bl}_{p} M} \mathbf{l}\left(h_{v}\right)\left(\overline{\mathbf{s}}_{\varepsilon}-\mathbf{s}\left(\omega_{\varepsilon}\right)\right) \omega_{\varepsilon}^{m} \\
= & \overline{\mathbf{s}}_{\varepsilon} \int_{\mathrm{Bl}_{p} M} \mathbf{l}\left(h_{v}\right) \omega_{\varepsilon}^{m}-\int_{\mathrm{Bl}_{p} M} \mathbf{l}\left(h_{v}\right) \mathbf{s}\left(\omega_{\varepsilon}\right) \omega_{\varepsilon}^{m} \\
= & \overline{\mathbf{s}}_{\varepsilon}\left(-\varepsilon^{2 m} h_{v}(p)-\frac{\varepsilon^{2 m+2}}{m+1} \Delta h_{v}(p)\right)-\int_{M} h_{v} \mathbf{s}(\omega) \omega^{m} \\
& \quad+2 \pi m(m-1) \varepsilon^{2 m-2} h_{v}(p)+2 \pi(m-2) \varepsilon^{2 m} \Delta h_{v}(p) \\
= & \operatorname{Fut}(M,[\omega], v)+A_{\varepsilon} h_{v}(p)+B_{\varepsilon} \Delta h_{v}(p) .
\end{aligned}
$$

Here

$$
\begin{gathered}
A_{\varepsilon}=2 \pi m(m-1) \varepsilon^{2 m-2}-\varepsilon^{2 m} \overline{\mathbf{s}}_{\varepsilon}, \\
B_{\varepsilon}=2 \pi(m-2) \varepsilon^{2 m}-\frac{\varepsilon^{2 m+2}}{m+1} \overline{\mathbf{s}}_{\varepsilon} .
\end{gathered}
$$

Using this together with the formula (40), we can obtain all the results that we are trying to prove.

We need one more result, relating the inner product of holomorphic Killing fields, introduced by Futaki-Mabuchi [13]. This inner product is simply the $L^{2}$ product of the Hamiltonian functions, which are normalized to have zero mean. So if $v, w$ have Hamiltonians $h_{v}, h_{w}$, normalized to have zero mean on $M$, then

$$
\langle v, w\rangle=\int_{M} h_{v} h_{w} \omega^{m} .
$$

If $v, w$ vanish at $p \in M$, then the product of the lifts to $\mathrm{Bl}_{p} M$ is given by

$$
\langle\hat{v}, \hat{w}\rangle=\int_{\mathrm{Bl}_{p} M} \mathbf{l}\left(h_{v}\right) \mathbf{l}\left(h_{w}\right) \omega_{\varepsilon}^{m}-\frac{1}{V_{\varepsilon}} \int_{\mathrm{Bl}_{p} M} \mathbf{l}\left(h_{v}\right) \omega_{\varepsilon}^{m} \int_{\mathrm{Bl}_{p} M} \mathbf{l}\left(h_{w}\right) \omega_{\varepsilon}^{m},
$$

where $V_{\varepsilon}$ is the volume of $\mathrm{Bl}_{p} M$ with respect to $\omega_{\varepsilon}$. The crucial property of this inner product is that it is independent of the representative $\omega_{\varepsilon}$ of its Kähler class.

Proposition 37. Assume that $\langle v, w\rangle=0$. Then for any $\delta>0$ we have on the blowup $\mathrm{Bl}_{p} M$, that

$$
\langle\hat{v}, \hat{w}\rangle=O\left(\varepsilon^{2 m-\delta}\right) .
$$

If in addition $h_{v}$ is normalized to have zero mean on $M$, and $h_{v}(p)=0$, then for any $\delta>0$ we have

$$
\langle\hat{v}, \hat{w}\rangle=O\left(\varepsilon^{2 m+2-\delta}\right) .
$$

In fact we could even take $\delta=0$ in both formulas, but we will not need this.

Proof. We can assume that $h_{v}$ and $h_{w}$ are normalized to have zero mean on $M$. Then from (35) we know that the averages of $\mathbf{l}\left(h_{v}\right)$ and $\mathbf{l}\left(h_{w}\right)$ on $\mathrm{Bl}_{p} M$ are of order $\varepsilon^{2 m}$, so in the formula (42) for $\langle\hat{v}, \hat{w}\rangle$ we can ignore the integrals of $\mathbf{l}\left(h_{v}\right)$ and $\mathbf{l}\left(h_{w}\right)$. 
Since $\mathbf{l}\left(h_{v}\right) \mathbf{l}\left(h_{w}\right)=h_{v} h_{w}$ and $\omega_{\varepsilon}=\omega$ outside $B_{2 r_{\varepsilon}}$, we just need to estimate the integrals on $B_{2 r_{\varepsilon}}$ with respect to the different metrics $\omega$ and $\omega_{\varepsilon}$. The volume of $B_{2 r_{\varepsilon}}$ is $O\left(r_{\varepsilon}^{2 m}\right)$ with respect to both $\omega$ and $\omega_{\varepsilon}$ so we obtain

$$
\langle\hat{v}, \hat{w}\rangle=O\left(r_{\varepsilon}^{2 m}\right) .
$$

If in addition $h_{v}(q)=0$, then we have $h_{v} \in C_{2}^{0}$, since also $\nabla h_{v}(q)=0$ by our assumption. It follows that also $\mathbf{l}\left(h_{v}\right) \in C_{2}^{0}$. This implies that

$$
\int_{B_{2 r_{\varepsilon}}} h_{v} h_{w} \omega^{m} \leqslant C \int_{0}^{2 r_{\varepsilon}} r^{2} r^{2 m-1} d r=O\left(r_{\varepsilon}^{2 m+2}\right),
$$

and also

$$
\int_{B_{2 r_{\varepsilon}}} h_{v} h_{w} \omega_{\varepsilon}^{m} \leqslant C\left(\int_{\varepsilon}^{2 r_{\varepsilon}} r^{2} r^{2 m-1} d r+\varepsilon^{2} \varepsilon^{2 m}\right)=O\left(r_{\varepsilon}^{2 m+2}\right) .
$$

We can do the construction with $r_{\varepsilon}=\varepsilon^{\alpha}$ for any $\alpha<1$, and choosing $\alpha$ sufficiently close to 1 we obtain the results we want. Note that one can do the analogous calculation algebro-geometrically and get a more precise result like in Proposition 35 but we will not need this.

We can now improve Proposition 34 following Stoppa [27] and 29] to get

Proposition 38. Suppose that $M$ admits an extremal metric $\omega$. Then $(M,[\omega])$ is relatively K-stable (with respect to smooth test-configurations).

The proof is essentially identical to the argument in 29, using Proposition 34 together with the formulas that we have shown in this section. In fact our situation is simpler since we are only dealing with test-configurations with smooth central fiber.

As an application we have the following proposition, which shows that we can only hope to construct extremal metrics on blowups $\mathrm{Bl}_{p} M$, for which $\nabla \mathbf{s}(\omega)$ vanishes at $p$.

Proposition 39. Suppose that $(M, \omega)$ is an extremal Kähler manifold, and $p \in M$ is such that $\nabla \mathbf{s}(\omega)$ does not vanish at $p$. Then $\left(\mathrm{Bl}_{p} M,\left[\omega_{\varepsilon}\right]\right)$ is relatively K-unstable for all sufficiently small $\varepsilon>0$.

Proof. Let $T \subset G_{p}$ be a maximal torus, with Lie algebra $\mathfrak{t}$. By our assumption, $\mathbf{s} \notin \mathbf{t}$. We have an orthogonal decomposition

$$
\mathbf{s}=\mathbf{s}^{\perp}+\mathbf{s}_{\mathfrak{t}},
$$

where $\mathbf{s}_{\mathfrak{t}} \in \mathfrak{t}$ and $\mathbf{s}^{\perp} \perp \mathfrak{t}$. Then $\nabla \mathbf{s}^{\perp}(p) \neq 0$, and we can assume that $\nabla \mathbf{s}^{\perp}$ generates a $\mathbf{C}^{*}$-action. If it did not, we could approximate $\mathbf{s}^{\perp}$ with elements of $\mathfrak{g}$ orthogonal to $\mathfrak{t}$, which do generate $\mathbf{C}^{*}$-actions.

Suppose then that $-\mathbf{s}^{\perp}$ generates the $\mathbf{C}^{*}$-action $\lambda(t)$, and let $q=\lim _{t \rightarrow 0} \lambda(t) \cdot p$. This way we obtain a test-configuration for $\left(\mathrm{Bl}_{p} M, \pi^{*}[\omega]-\varepsilon^{2}[E]\right)$ with central fiber $\mathrm{Bl}_{q} M$. The Futaki invariant of the test-configuration is given by

$$
\operatorname{Fut}\left(\mathrm{Bl}_{q} M, \pi^{*}[\omega]-\varepsilon^{2}[E],-\hat{\mathbf{s}}^{\perp}\right)=\operatorname{Fut}\left(M,[\omega],-\mathbf{s}^{\perp}\right)+O\left(\varepsilon^{2 m-2}\right),
$$

using a calculation similar to Corollary 36 , Since

$$
\operatorname{Fut}\left(M,[\omega],-\mathbf{s}^{\perp}\right)=\left\langle-\mathbf{s}^{\perp}, \mathbf{s}\right\rangle=-\left\|\mathbf{s}^{\perp}\right\|^{2},
$$


we have a constant $c_{0}>0$ such that

$$
\operatorname{Fut}\left(\mathrm{Bl}_{q} M, \pi^{*}[\omega]-\varepsilon^{2}[E],-\hat{\mathbf{s}}^{\perp}\right)<-c_{0},
$$

for sufficiently small $\varepsilon$. In order to show that $\left(\mathrm{Bl}_{p} M, L_{\varepsilon}\right)$ is relatively $\mathrm{K}$-unstable, we still need to adjust this test-configuration to be orthogonal to $\mathbf{t}$. Since $\mathbf{s}^{\perp}$ is orthogonal to $\mathfrak{t}$, it follows from Proposition 37 that for any $v \in \mathfrak{t}$ we have

$$
\left\langle\hat{\mathbf{s}}^{\perp}, v\right\rangle=O\left(\varepsilon^{2 m-\delta}\right),
$$

for any $\delta>0$. This means that after modifying the test-configuration with an element in $\mathfrak{t}$ to make it orthogonal to $\mathfrak{t}$ on $\mathrm{Bl}_{q} M$, the Futaki invariant will still be negative for sufficiently small $\varepsilon$. It follows that $\left(\mathrm{Bl}_{p} M, L_{\varepsilon}\right)$ is relatively $\mathrm{K}$-unstable for sufficiently small $\varepsilon>0$.

4.3. Test-configurations for blowups. In this section we will give the proof of Theorem 2. Suppose that $(M, \omega)$ is $\operatorname{cscK}$, and suppose that $v$ is a Hamiltonian holomorphic vector field on $M$ generating a $\mathbf{C}^{*}$-action $\lambda(t)$. Then for any $p \in M$, $\lambda(t)$ induces a test-configuration for $\left(\mathrm{Bl}_{p} M,\left[\omega_{\varepsilon}\right]\right)$. The total space of this testconfiguration is simply the blowup of the product $M \times \mathbf{C}$ along the closure of the orbit

$$
\left\{(\lambda(t) \cdot p, t): t \in \mathbf{C}^{*}\right\} .
$$

If $q=\lim _{t \rightarrow 0} \lambda(t) \cdot p$, then the central fiber of the test-configuration is $\left(\mathrm{Bl}_{q} M, \omega_{\varepsilon}\right)$. The induced $\mathbf{C}^{*}$-action on $\mathrm{Bl}_{q} M$ is given by $\lambda(t)$, which lifts to $\mathrm{Bl}_{q} M$ since $q$ is a fixed point. The formula (39) can be used to compute the Futaki invariant of this test-configuration.

When combined with Theorem 1, the following proposition implies Theorem 2 , This proposition generalizes [32, Theorem 5] to Kähler manifolds.

Proposition 40. Suppose that $n>2$, and for some $\varepsilon_{0}>0$ there does not exist $q \in G^{c} \cdot p$ with $\mu(q)+\varepsilon \Delta \mu(q)=0$ for any $\varepsilon \in\left(0, \varepsilon_{0}\right)$. Then $\left(\mathrm{Bl}_{p} M,\left[\omega_{\varepsilon}\right]\right)$ is $K$-unstable for all sufficiently small $\varepsilon>0$.

Proof. By moving $p$ in its $G^{c}$-orbit, we can assume that $G_{p}$ is a maximal compact subgroup of $G_{p}^{c}$. Then if $T \subset G_{p}$ is a maximal torus, then $T^{c} \subset G_{p}^{c}$ is also a maximal torus. As in Section 2 we will work with the group $G_{T^{\perp}}$. The corresponding moment map $\mu_{T^{\perp}}$ is simply the orthogonal projection of $\mu$ onto $\mathfrak{g}_{T^{\perp}}$. Our assumption says that $p$ is unstable for the action of $G_{T^{\perp}}^{c}$ with respect to the moment map

$$
\mu_{T^{\perp}}+\varepsilon \Delta \mu_{T^{\perp}}
$$

for all sufficiently small $\varepsilon>0$. There are several cases to consider separately.

- Suppose that $p$ is strictly unstable for the moment map $\mu_{T \perp}$. This means that there is a $v \in \mathfrak{g}_{T^{\perp}}$ generating a $\mathbf{C}^{*}$-action $\lambda(t)$, such that

$$
\lim _{t \rightarrow 0}\langle\mu(\lambda(t) \cdot p), v\rangle<0 .
$$

Write $q=\lim _{t \rightarrow 0} \lambda(t) \cdot p$. We then have $h_{v}(q)<0$. From Corollary 36 it follows that the corresponding test-configuration for $\left(\mathrm{Bl}_{p} M,\left[\omega_{\varepsilon}\right]\right)$ has Futaki invariant

$$
\operatorname{Fut}\left(\mathrm{Bl}_{q} M,\left[\omega_{\varepsilon}\right], \hat{v}\right)<-c_{0} \varepsilon^{2 m-2},
$$

for some $c_{0}>0$. This means that $\left(\mathrm{Bl}_{p} M,\left[\omega_{\varepsilon}\right]\right)$ is K-unstable. 
- Suppose that $p$ is semistable for the moment map $\mu_{T^{\perp}}$, and we can find a $v \in \mathfrak{g}_{T^{\perp}}$ generating a $\mathbf{C}^{*}$-action $\lambda(t)$, such that

$$
\begin{gathered}
\lim _{t \rightarrow 0}\langle\mu(\lambda(t) \cdot p), v\rangle=0 \\
\lim _{t \rightarrow 0}\langle\Delta \mu(\lambda(t) \cdot p), v\rangle<0 .
\end{gathered}
$$

Writing again $q=\lim _{t \rightarrow 0} \lambda(t) \cdot p$ we then have $h_{v}(q)=0$ and $\Delta h_{v}(q)<0$. From Corollary 36 we have

$$
\operatorname{Fut}\left(\mathrm{Bl}_{q} M,\left[\omega_{\varepsilon}\right], \hat{v}\right)<-c_{0} \varepsilon^{2 m},
$$

for some $c_{0}>0$, and so it follows that $\left(\mathrm{Bl}_{p} M,\left[\omega_{\varepsilon}\right]\right)$ is K-unstable.

- In the remaining case $p$ is semistable with respect to $\mu_{T^{\perp}}+\varepsilon \Delta \mu_{T^{\perp}}$ for all sufficiently small $\varepsilon>0$. This implies that we can find $q_{\varepsilon}$ in the boundary $\partial G^{c} \cdot p$ of the $G^{c}$-orbit such that

$$
\mu_{T^{\perp}}\left(q_{\varepsilon}\right)+\varepsilon \Delta \mu_{T^{\perp}}\left(q_{\varepsilon}\right)=0 .
$$

Since $\partial G^{c} \cdot p$ is a finite union of orbits, at least one orbit must contain infinitely many $q_{\varepsilon}$. Choose $q_{\varepsilon_{1}}$ and $q_{\varepsilon_{2}}$ to be in the same orbit. Since the moment maps are equivariant and $T$ fixes $q_{\varepsilon_{i}}$, we have

$$
\mu\left(q_{\varepsilon_{i}}\right)+\varepsilon_{i} \Delta \mu\left(q_{\varepsilon_{i}}\right) \in \mathfrak{t} .
$$

In addition, the projection of the moment map to the stabilizer is an invariant of the orbit, so if $q$ is in the same $G_{T^{\perp}}^{c}$ orbit as the $q_{\varepsilon_{i}}$, then

$$
\operatorname{pr}_{\mathfrak{g}_{q}}\left(\mu(q)+\varepsilon_{i} \Delta \mu(q)\right) \in \mathfrak{t} .
$$

Since this holds for at least two different $\varepsilon_{i}$, we must have

$$
\operatorname{pr}_{\mathfrak{g}_{q}} \mu(q), \operatorname{pr}_{\mathfrak{g}_{q}} \Delta \mu(q) \in \mathfrak{t} .
$$

It follows that the stabilizer of $q_{\varepsilon_{1}}$ in $G_{T^{\perp}}^{c}$ is reductive and so there is a local slice for the action of $G_{T^{\perp}}^{c}$ near $q_{\varepsilon_{1}}$ (see Sjamaar [25] or Snow [26]). Using the Hilbert-Mumford criterion applied to the action of the stabilizer on the tangent space at $q_{\varepsilon_{1}}$, we can find a $v \in \mathfrak{g}_{T^{\perp}}$ generating a $\mathbf{C}^{*}$-action $\lambda(t)$, and a point $p^{\prime} \in G^{c} \cdot p$, such that $q_{\varepsilon_{1}}=\lim _{t \rightarrow 0} \lambda(t) \cdot p^{\prime}$. This means that there exists a test-configuration for $\left(\mathrm{Bl}_{p} M,\left[\omega_{\varepsilon}\right]\right)$, whose central fiber is $\left(\mathrm{Bl}_{q} M,\left[\omega_{\varepsilon}\right]\right)$, writing $q=q_{\varepsilon_{1}}$.

We claim that this test-configuration has zero Futaki invariant. Indeed, since $v \in \mathfrak{t}^{\perp}$, it follows from (44) that the Hamiltonian $h_{v}$ satisfies $h_{v}(q)=\Delta h_{v}(q)=0$. In addition since $\mathbf{s} \in \mathfrak{t}$, we have $\operatorname{Fut}(M,[\omega], v)=0$. Corollary 36 then implies that $\operatorname{Fut}\left(\mathrm{Bl}_{q} M,\left[\omega_{\varepsilon}\right], \hat{v}\right)=0$. It follows that $\left(\mathrm{Bl}_{p} M,\left[\omega_{\varepsilon}\right]\right)$ is $\mathrm{K}$-unstable.

Combining our results we can prove Theorem 2 ,

Proof of Theorem Q (1) $\Rightarrow(2)$ : This follows from Proposition 38

$(2) \Rightarrow(3)$ : This is the statement of Proposition 40.

$(3) \Rightarrow(1)$ : It follows from Theorem 1], that under the assumption the blowup $\mathrm{Bl}_{p} M$ admits an extremal metric in the class $\left[\omega_{\varepsilon}\right]$. We just need to check that this metric has constant scalar curvature. To do this we need to compute the Futaki invariant $\operatorname{Fut}\left(\mathrm{Bl}_{p} M,\left[\omega_{\varepsilon}\right], \hat{v}\right)$ for all $v \in \mathfrak{g}_{p}$. Since $(M, \omega)$ is $\operatorname{cscK}$ we know that 
$\operatorname{Fut}(M,[\omega], v)=0$. In addition if $\varepsilon$ is sufficiently small, then an argument similar to the proof of Proposition 7 shows that

$$
\operatorname{pr}_{\mathfrak{g}_{p}} \mu(p), \operatorname{pr}_{\mathfrak{g}_{p}} \Delta \mu(p)=0 .
$$

Therefore $h_{v}(p)=\Delta h_{v}(p)=0$, so from Corollary 36 we get $\operatorname{Fut}\left(\mathrm{Bl}_{p} M,\left[\omega_{\varepsilon}\right], \hat{v}\right)=0$. It follows that the extremal metric constructed using Theorem 1 has constant scalar curvature.

\section{REFERENCES}

[1] C. Arezzo and F. Pacard. Blowing up and desingularizing constant scalar curvature Kähler manifolds. Acta Math., 196(2):179-228, 2006.

[2] C. Arezzo and F. Pacard. Blowing up Kähler manifolds with constant scalar curvature II. Ann. of Math. (2), 170(2):685-738, 2009.

[3] C. Arezzo, F. Pacard, and M. A. Singer. Extremal metrics on blow ups. Duke Math. J., 157(1):1-51, 2011.

[4] O. Biquard and Y. Rollin. Smoothing singular extremal Kähler surfaces and minimal lagrangians. arXiv:1211.695\%.

[5] S. Bochner and W. T. Martin. Several complex variables, volume 10 of Princeton Mathematical Series. Princeton University Press, Princeton, N.J., 1948.

[6] E. Calabi. Extremal Kähler metrics. In S. T. Yau, editor, Seminar on Differential Geometry. Princeton, 1982.

[7] E. Calabi. Extremal Kähler metrics II. In Differential geometry and complex analysis, pages 95-114. Springer, 1985.

[8] X. X. Chen and G. Tian. Geometry of Kähler metrics and foliations by holomorphic discs. Publ. Math. Inst. Hautes Études Sci., (107):1-107, 2008.

[9] A. Clarke and C. Tipler. Lower bounds on the modified K-energy and complex deformations. preprint.

[10] A. Della Vedova. CM-stability of blow-ups and canonical metrics. preprint (2008).

[11] S. K. Donaldson. Scalar curvature and stability of toric varieties. J. Differential Geom., 62:289-349, 2002

[12] A. Futaki. An obstruction to the existence of Einstein-Kähler metrics. Invent. Math., 73:437443, 1983.

[13] A. Futaki and T. Mabuchi. Bilinear forms and extremal Kähler vector fields associated with Kähler classes. Math. Ann., 301:199-210, 1995.

[14] P. Gauduchon. Invariant scalar-flat Kähler metrics on $\mathcal{O}(-l)$. preprint, 2012.

[15] F. C. Kirwan. Cohomology of Quotients in Symplectic and Algebraic Geometry. Princeton University Press, 1984.

[16] C. LeBrun. Counter-examples to the generalized positive action conjecture. Comm. Math. Phys., 118(4):591-596, 1988.

[17] C. LeBrun and S. R. Simanca. Extremal Kähler metrics and complex deformation theory. Geom. and Func. Anal., 4(3):298-336, 1994.

[18] C. LeBrun and M. A. Singer. Existence and deformation theory for scalar-flat Kähler metrics on compact complex surfaces. Invent. Math., 112(2):273-313, 1993.

[19] H. Li and Y. Shi. The Futaki invariant on the blowup of Kähler surfaces. arXiv:1211.2954.

[20] I. Mundet i Riera. A Hitchin-Kobayashi correspondence for Kähler fibrations. J. Reine Angew. Math., 528:41-80, 2000.

[21] F. Pacard. Constant scalar curvature and extremal Kähler metrics on blow ups. In Proceedings of the International Congress of Mathematicians. Volume II, pages 882-898, New Delhi, 2010. Hindustan Book Agency.

[22] F. Pacard and $\mathrm{X}$. Xu. Constant mean curvature spheres in Riemannian manifolds. Manuscripta Math., 128(3):275-295, 2009.

[23] Y. Rollin and M. A. Singer. Non-minimal scalar-flat Kähler surfaces and parabolic stability. Invent. Math., 162(2):235-270, 2005.

[24] S. R. Simanca. Kähler metrics of constant scalar curvature on bundles over $C \mathrm{P}_{n-1}$. Math. Ann., 291(2):239-246, 1991. 
[25] R. Sjamaar. Holomorphic slices, symplectic reduction and multiplicities of representations. Ann. of Math. (2), 141(1):87-129, 1995.

[26] D. M. Snow. Reductive group actions on Stein spaces. Math. Ann., 259(1):79-97, 1982.

[27] J. Stoppa. K-stability of constant scalar curvature Kähler manifolds. Adv. Math., 221(4):1397$1408,2009$.

[28] J. Stoppa. Unstable blowups. J. Algebraic Geom., 19(1):1-17, 2010.

[29] J. Stoppa and G. Székelyhidi. Relative K-stability of extremal metrics. J. Eur. Math. Soc., 13(4):899-909, 2011.

[30] G. Székelyhidi. Extremal metrics and K-stability. PhD thesis, Imperial College, London, 2006.

[31] G. Székelyhidi. Extremal metrics and K-stability. Bull. Lond. Math. Soc., 39(1):76-84, 2007.

[32] G. Székelyhidi. On blowing up extremal Kähler manifolds. Duke Math. J., 161(8):1411-1453, 2012.

[33] A. Teleman. Symplectic stability, analytic stability in non-algebraic complex geometry. Internat. J. Math., 15(2):183-209, 2004.

[34] G. Tian. Kähler-Einstein metrics with positive scalar curvature. Invent. Math., 137:1-37, 1997.

[35] C. Tipler. Extremal Kähler metrics on blow-ups of parabolic ruled surfaces. arXiv:1104.4315.

[36] V. Tosatti. The K-energy on small deformations of constant scalar curvature Kähler manifolds, volume 21 of Advanced Lectures in Math., pages 139-150. International Press, 2012.

[37] S.-T. Yau. Open problems in geometry. Proc. Symposia Pure Math., 54:1-28, 1993.

Department of Mathematics, University of Notre Dame, Notre Dame, IN 46615

E-mail address: gszekely@nd.edu 